\title{
Imposing periodic boundary condition on arbitrary meshes by polynomial interpolation
}

\author{
V.-D. Nguyen ${ }^{\mathrm{a}}$, E. Béchet ${ }^{\mathrm{a}}$, C. Geuzaine ${ }^{\mathrm{b}}$, L. Noels ${ }^{\mathrm{a}, *}$ \\ ${ }^{a}$ University of Liège, \\ Department of Aerospace and Mechanical Engineering Department, \\ Chemin des Chevreuils 1, B-4000 Liège, Belgium \\ ${ }^{b}$ University of Liége, \\ Department of Electrical Engineering and Computer Science \\ B28 P32, B-4000 Liège, Belgium
}

\begin{abstract}
In order to predict the effective properties of heterogeneous materials using the finite element approach, a boundary value problem (BVP) may be defined on a representative volume element (RVE) with appropriate boundary conditions, among which periodic boundary condition is the most efficient in terms of convergence rate. The classical method to impose the periodic boundary condition requires the identical meshes on opposite RVE boundaries. This condition is not always easy to satisfy for arbitrary meshes. This work develops a new method based on polynomial interpolation that avoids the need of matching mesh condition on opposite RVE boundaries.
\end{abstract}

Keywords: Polynomial interpolation, Periodic condition, FEM, Computational homogenization, Heterogeneous materials

\section{Introduction}

The behavior of heterogeneous materials depends mainly on the material properties, volume fraction and spatial distribution of the different constituents, which led to numerous developments to link the macroscopic properties to the details of the material micro-structure. As the increase of material requirements in high performance applications provides a strong stimulus for a new material design, which can be achieved by manipulating the micro-structure, proper evaluation of this heterogeneous material behavior is mandatory.

The effective or macroscopic properties of heterogeneous materials, have traditionally been obtained by homogenization techniques based on the analytical or semi-analytical formulation (for instance, mean field homogenization [1]). In recent years, an alternative approach, so-called computational homogenization, has been developed, mainly to account for non-linear behaviors at the micro-scale (See the references by Michel et al. [2], Miehe et al. [3], Kouznetsova et al. [4, 5, 6], Kaczmarczyk et al. [7], Kanouté et al. [8], Peric et al. [9], Geers et al. [10], as a nonexhaustive list). The basic idea of the computational homogenization techniques is to obtain the effective properties of heterogeneous materials by solving the boundary value problem with appropriate boundary conditions defined on a representative volume element (RVE), which contains all necessary information of the micro-structure. As the RVE

${ }^{*}$ Corresponding author; Phone: +32 4366 4826; Fax: +32 43669505

Email address: L. Noels@ulg. ac.be (L. Noels) 
size should be large enough to represent statistically the micro-structure, and as the computation time increases with the RVE size, it is important to provide an analytical or numerical method to determine the critical size of the RVE, as proposed by Kanit et al. [11] for random composites.

When considering computational homogenization, the effective stress tensor and tangent operator related to the strain tensor at any point of the macroscopic level are computed by modeling in details, e.g. with the finite element method, the boundary value problem associated with the micro-structure at that point. This technique does not require a constitutive macroscopic assumption on the macroscopic level, but the boundary conditions of the micro problem have to be correctly defined. Among the three classical boundary conditions proposed: (1) linear displacement boundary condition (Dirichlet condition); (2) constant traction boundary condition (Neumann condition) and (3) periodic boundary condition, many numerical studies $[11,12,13]$ show that the periodic boundary condition is the most efficient in terms of convergence rate when the RVE size increases, as presented in Fig. 1. For the three kinds of boundary conditions, the increase of the RVE size leads to a better estimation of the effective properties, but for a given RVE size, the periodic boundary condition provides a better estimation than the linear displacement or than the constant traction boundary conditions. Although rigorous for periodic structures, this conclusion also holds if the micro-structure does not possess geometrical periodicity $[11,12,13]$. Because of its efficiency, this work takes an interest in applying the periodic boundary condition on arbitrary meshes.

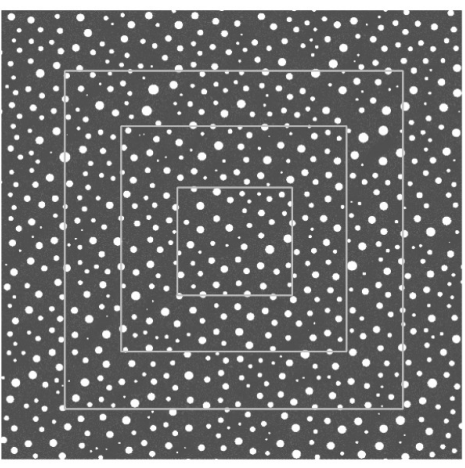

(a)

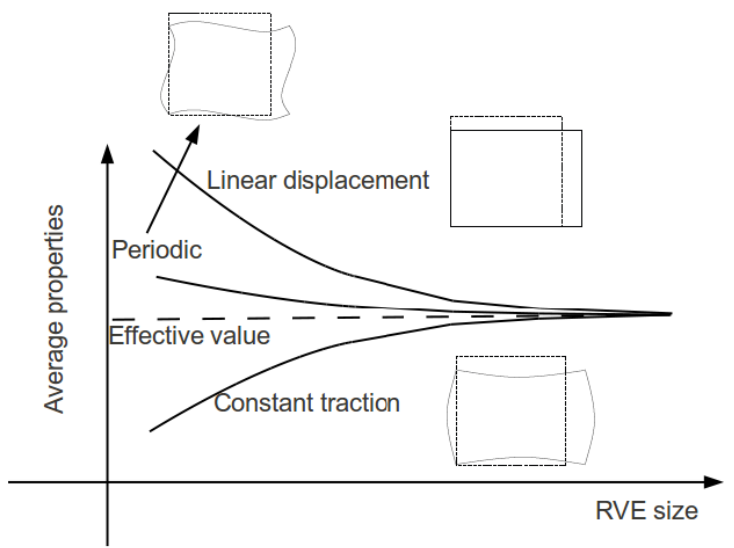

(b)

Figure 1: Illustration of the convergence withe RVE size following [12]: (a) Representative volume element (RVE) with different sizes and (b) Convergence of average properties with increasing RVE size for different boundary condition types: linear displacement, constant traction and periodic boundary conditions.

To apply the periodic boundary condition, the classical method consists in enforcing the same value for degrees of freedom of matching nodes on two opposite RVE sides. Thus, it requires a periodic mesh, which has the same mesh distribution on two opposite parts of the RVE boundary. The enforcement of unique values for the degrees of freedom of matching nodes can then be done with the Lagrange multiplier method $[2,3,7]$ or directly by constraint eliminations $[2,9]$. In a more general setting, the conformity of mesh distributions on opposite boundaries of RVE cannot always be guaranteed, leading to a non-periodic mesh. For instance, in studies analyzing real micro-structures where the mesh is obtained by converting the finite element model from a micro-structure image processing [14], the mesh is generally non-periodic.

Thus it is necessary to find another method which does not need a periodic mesh in order to apply the periodic boundary condition. For this purpose, Yuan et al. [15] proposed a master/slave approach for imposing periodic boundary condition for non-matching meshes. Larsson et al. [13] developed a weak enforcement of the periodic boundary condition by introducing independently the finite element discretization of traction boundary and by allowing for the transition between the strongest form (periodic boundary condition) to the weakest form (Neumann condition). Tyrus et al. [16] implemented the periodic boundary condition for periodic composite materials, for arbitrary non-periodic mesh by enforcing a linear displacement field at intersection of fibers and RVE sides and a cubic displacement field at 
intersection of matrix and RVE sides. Although efficient, this method requires a priori knowledge of the deformation shape of the RVE and is thus only applicable to a periodic 2D unit-cell (composite fibers located solely at the corners of the 2D-RVE).

In this work, it is proposed to enhance the method of Tyrus et al. [16] by considering new general functions describing the boundary deformation, without requiring a priori knowledge of the deformed shape, of the material structure or of the mesh distribution (periodic or non-periodic). In this new method, the displacement field of two opposite RVE sides is interpolated by linear combinations of some shape functions. The degrees of freedom of two opposite RVE sides are then substituted by the coefficients of these shape functions. This method is general in the 2-dimensional and 3-dimensional cases for periodic or random materials. For simplicity and efficiency, the two polynomial interpolations are chosen as interpolant: the Lagrange interpolation using the Lagrange shape functions and the cubic spline interpolation using the Hermite shape functions. This method allows to enforce strongly the periodic boundary condition, without requirement of periodic mesh, from the "weakest constraint" (linear displacement boundary condition) corresponding to the polynomial order 1 to the "strongest constraint" (periodic boundary condition) corresponding to the infinite polynomial order. Although this last case is theoretical, it can be approximated by using an interpolated function of high degree. The proposed method is shown to be easy to implement, and allows extracting effective material properties of a heterogeneous structures using a RVE of reduced size, for periodic and non-periodic structures.

The paper is organized as follows. Section 2 recalls the main preliminaries of the computational homogenization method in sub-scale modeling. Section 3 introduces the theory of the new polynomial interpolation method for periodic boundary condition, and section 4 its finite element implementation. The sensibility study on user parameters, such as the polynomial degree, is studied in section 5. Convergence of the effective material properties extracted from the model when increasing the RVE size is illustrated in section 6 for periodic and non-periodic structures. In these two sections, the results obtained on 2D-RVE with the new method are compared with those given by the elimination constraint method and the linear displacement boundary condition. Finally, in order to demonstrate the efficiency of the method, section 7 presents the results for 3-dimensional cases with periodic and non-periodic meshes.

\section{Sub-scale modeling and boundary condition}

In order to estimate the effective properties of heterogeneous materials, a classical approach to account for the effect of micro-heterogeneity is to implement the sub-scale modeling based on the concept of a local representative volume element (RVE) with appropriate boundary conditions. This concept is based on the assumption that any material point $\boldsymbol{X} \in \bar{V}$ in the continuum scale is associated to a local RVE whose domain is $V$ with boundary $\partial V$, see Fig. 2, the characteristic length of the sub-scale problem being much smaller than the characteristic length of the continuum scale. In this section, the preliminaries of sub-scale modeling are presented.

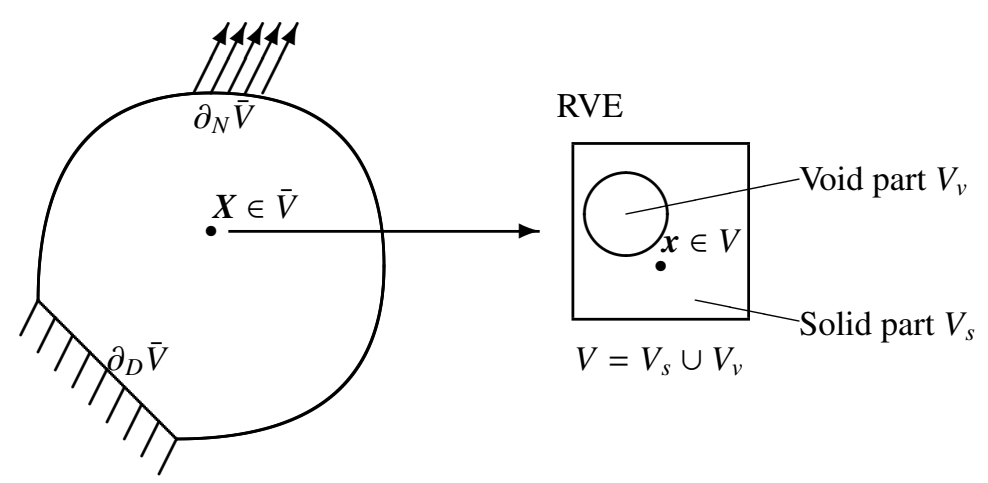

Figure 2: Continuum macro-structure associated to a representative volume element. 


\subsection{RVE equilibrium state}

In absence of body forces, assuming the RVE is subjected to an external traction $\overline{\boldsymbol{t}}$ via its boundary $\partial V$, the equilibrium state is governed by the equilibrium equations

$$
\begin{aligned}
\frac{\partial \sigma_{i j}}{\partial x_{j}} & =0 \quad \forall \boldsymbol{x} \in V, \text { and } \\
\sigma_{i j} n_{j} & =\bar{t}_{i} \quad \forall \boldsymbol{x} \in \partial V,
\end{aligned}
$$

where $\sigma$ is the Cauchy stress tensor. The mechanical property of the micro-constituents is described through a constitutive law, and for the hyperelastic material, the stress tensor $\sigma$ is related to the strain tensor $\epsilon$ via the strain energy function $W$ :

$$
\sigma_{i j}=\frac{\partial W}{\partial \varepsilon_{i j}} .
$$

The equilibrium state of the RVE is assumed to be consistent with the boundary condition, which are related to the macroscopic strain field $\overline{\boldsymbol{\varepsilon}}$.

\subsection{Averaging theory}

The macro-scale properties, macro-stress tensor $\bar{\sigma}$ and macro-strain tensor $\bar{\varepsilon}$, are defined as the volume average of the sub-scale stress and strain tensors over the RVE [3, 9]:

$$
\begin{aligned}
\bar{\sigma}_{i j} & =\frac{1}{V} \int_{V} \sigma_{i j} d V, \text { and } \\
\bar{\varepsilon}_{i j} & =\frac{1}{V} \int_{V} \varepsilon_{i j} d V .
\end{aligned}
$$

Still in absence of body forces, applying the Gauss theorem allows rewriting these two expressions as

$$
\begin{aligned}
\bar{\sigma}_{i j} & =\frac{1}{V} \oint_{\partial V} t_{i} x_{j} d S, \text { and } \\
\bar{\varepsilon}_{i j} & =\frac{1}{V} \oint_{\partial V} \frac{1}{2}\left(u_{i} n_{j}+u_{j} n_{i}\right) d S^{1} .
\end{aligned}
$$

\subsection{Hill-Mandel principle}

For the sub-scale-modeling to be energetically consistent, the deformation energy at the macroscopic level should be equal to the volume average of micro-scale stress work $[3,9]$. It means that at any equilibrium state of the RVE characterized by the stress field $\sigma$ and the strain field $\varepsilon$, the following equation must be satisfied

$$
\bar{\sigma}_{i j} \bar{\varepsilon}_{i j}=\frac{1}{V} \int_{V} \sigma_{i j} \varepsilon_{i j} d V .
$$

As in the absence of body forces

$$
\frac{1}{V} \oint_{\partial V} t_{i} u_{i} d S=\frac{1}{V} \int_{V} \sigma_{i j} \varepsilon_{i j} d V
$$

\footnotetext{
${ }^{1}$ As pointed out by Michel et al. [2], this last relation requires particular attention when voids are present and intersect the volume boundary. If the volume $V=V_{S} \cup V_{V}$ is the union of the solid volume $V_{S}$ and of the voids volume $V_{V}$, one has $\bar{\varepsilon}_{i j}=\frac{1}{V} \int_{V_{S}} \cup V_{V} \varepsilon_{i j} d V$. When applying the Gauss theorem on both $V_{V}$ and $V_{S}$, as their respective surface normals on solid/void interfaces are in opposite direction, and as the surface normal on the intersection of voids with the boundary is in the same direction as the normal of surface $\partial V$ the expression simplifies in Eq. (7). The same development can be done for Eq. (6).
} 
and as Eqs. (4-7) imply successively

$$
\begin{aligned}
\frac{1}{V} \oint_{\partial V} t_{i} \bar{\varepsilon}_{i k} x_{k} d S & =\bar{\varepsilon}_{i k} \frac{1}{V} \int_{\partial V} t_{i} x_{k} d S=\bar{\varepsilon}_{i j} \bar{\sigma}_{i j}, \\
\frac{1}{V} \oint_{\partial V} u_{i} \bar{\sigma}_{i j} n_{j} d S & =\bar{\sigma}_{i j} \frac{1}{V} \int_{\partial V} n_{j} u_{i} d S=\bar{\varepsilon}_{i j} \bar{\sigma}_{i j}, \text { and } \\
\frac{1}{V} \oint_{\partial V} n_{j} \bar{\varepsilon}_{i j} \bar{\sigma}_{i k} x_{k} d S & =\bar{\varepsilon}_{i j} \bar{\sigma}_{i k} \frac{1}{V} \int_{\partial V} n_{j} x_{k} d S=\bar{\varepsilon}_{i j} \bar{\sigma}_{i j},
\end{aligned}
$$

combining Eqs. (9-12) allows rewriting the Hill-Mandel principle (8) as

$$
\oint_{\partial V}\left(t_{i}-\bar{\sigma}_{i j} n_{j}\right)\left(u_{i}-\bar{\varepsilon}_{i k} x_{k}\right) d S=0 .
$$

Thus, in order to fulfill the Hill-Mandel principle when analyzing the sub-scale problem, the boundary condition must be applied in such a way that Eq. (13) is a priori satisfied.

Without loss of generality, the microscopic displacement field on the RVE boundary $(\boldsymbol{u})$ can be decomposed into two parts: the mean part $(\overline{\boldsymbol{u}})$ and the zero-mean fluctuation part $(\tilde{\boldsymbol{u}})$ :

$$
u_{i}=\bar{u}_{i}+\tilde{u}_{i}=\bar{\varepsilon}_{i j} x_{j}+\tilde{u}_{i}
$$

where the fluctuation strain field is given by

$$
\tilde{\varepsilon}_{i j}=\frac{1}{2}\left(\frac{\partial \tilde{u}_{i}}{\partial x_{j}}+\frac{\partial \tilde{u}_{j}}{\partial x_{i}}\right)
$$

These definitions allows to express the Hill-Mandel condition (13) in the more convenient form

$$
\frac{1}{V} \oint_{\partial V} t_{i} \tilde{u}_{i} d S=0
$$

\subsection{RVE boundary condition}

As previously stated, the boundary condition on RVE for the displacement field $\tilde{\boldsymbol{u}}$ and the traction field $\boldsymbol{t}$ should be defined in order to satisfy (13) or (16). As illustrated on Fig. 1, three types of boundary conditions are generally considered:

Constant traction boundary condition (Neumann boundary condition): the traction field on the RVE boundary $\partial V$ is prescribed in terms of the macroscopic stress:

$$
t_{i}=\bar{\sigma}_{i j} n_{j} \quad \forall x \in \partial V
$$

which satisfies (13).

Linear displacement boundary condition (Dirichlet boundary condition): the displacement field on the RVE boundary is constrained in terms of the macroscopic strain:

$$
\tilde{u}_{i}=u_{i}-\bar{\varepsilon}_{i j} x_{j}=0 \quad \forall \boldsymbol{x} \in \partial V,
$$

which also satisfies (13).

Periodic boundary condition: the RVE boundary $\partial V$ is decomposed into two parts: a positive part $\partial V^{+}$and a negative part $\partial V^{-}$with $\partial V^{+} \cup \partial V^{-}=\partial V, \partial V^{+} \cap \partial V^{-}=\emptyset$, and with the associated outward normal $\boldsymbol{n}^{+}=-\boldsymbol{n}^{-}$at corresponding points $\boldsymbol{x}^{+} \in \partial V^{+}$and $\boldsymbol{x}^{-} \in \partial V^{-}$, respectively, see Fig. 3. The periodic boundary condition represents the periodicity of fluctuations field and anti-periodicity of the traction field on RVE boundary:

$$
\begin{array}{rll}
\tilde{\boldsymbol{u}}\left(\boldsymbol{x}^{+}\right)= & \tilde{\boldsymbol{u}}\left(\boldsymbol{x}^{-}\right) & \forall \boldsymbol{x}^{+} \in \partial V^{+} \text {and matching } \boldsymbol{x}^{-} \in \partial V^{-}, \\
\boldsymbol{t}\left(\boldsymbol{x}^{+}\right)= & -\boldsymbol{t}\left(\boldsymbol{x}^{-}\right) & \forall \boldsymbol{x}^{+} \in \partial V^{+} \text {and matching } \boldsymbol{x}^{-} \in \partial V^{-} .
\end{array}
$$




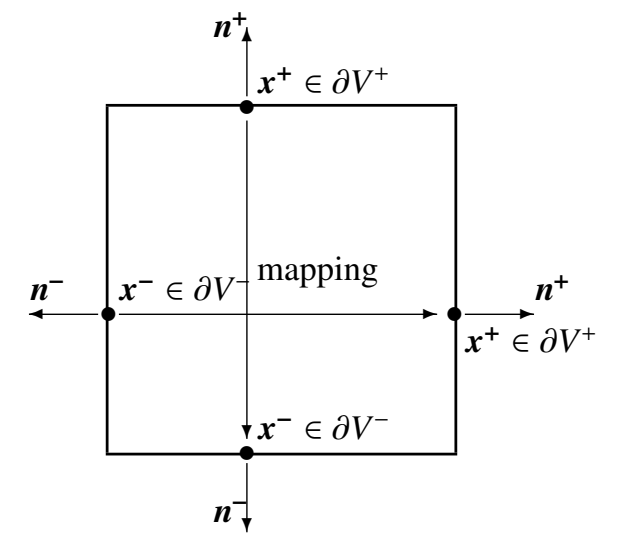

Figure 3: Node mapping to apply the periodic boundary condition.

Many numerical studies show that the periodic boundary condition is the most efficient choice, even if the arrangement of the micro-structure is non-periodic $[11,12,13]$. So, the periodic boundary condition is widely used for estimating the effective properties of heterogeneous materials. However, in the context of the finite element method, two mesh types can be found:

- Periodic mesh: this mesh type is characterized by the same mesh distribution on the positive part and on the negative part of the RVE boundary. It is trivial to impose periodic constraints by using directly the periodic condition (19). These linear constraints can be introduced by the constraint elimination method $[2,9]$ or the Lagrange multiplier method $[2,3,7]$;

- Non- periodic mesh: in more general cases, the same distribution of nodes on opposite parts of the RVE boundary does not hold. This is especially true in cases where the mesh results from imaging technique as for the randomly foams. Thus for a node on a boundary there is not always a conforming node on the opposite side: the mesh is non-periodic. For this mesh type, applying periodic boundary condition is not trivial, and the aim of this work is to develop an efficient method to enforce the periodic boundary condition for a general mesh design.

\subsection{Extraction of effective properties}

The purpose of studying the RVE at the micro-scale is to extract the macro-stress tensor $\overline{\boldsymbol{\sigma}}(4)$. For elastic materials, the average tangent moduli $\mathbb{C}$ is defined by the linear relation of the macro-stress tensor $\bar{\sigma}$ and the macro-strain tensor $\bar{\varepsilon}(5)$ :

$$
\bar{\sigma}_{i j}=\mathbb{C}_{i j k l} \bar{\varepsilon}_{k l},
$$

resulting into a macro-strain energy on the RVE given by:

$$
U=\frac{V}{2} \bar{\sigma}_{i j} \bar{\varepsilon}_{i j}=\frac{V}{2} \bar{\varepsilon}_{i j} \mathbb{C}_{i j k l} \bar{\varepsilon}_{k l} .
$$

The sub-scale model is solved by applying the macro-strain tensor $\bar{\varepsilon}$ through boundary conditions (14) to the RVE, resulting into a BVP solved using the finite element method. In case of choosing the periodic boundary condition kind, relations (19)-(20) should be enforced. By approximating the relations (4), the macro-stress tensor can be directly estimated via the integral:

$$
\bar{\sigma}_{i j}=\frac{1}{V} \int_{V} \sigma_{i j} d V=\frac{1}{V} \sum_{e=1}^{N_{e l e}} \sum_{p t=1}^{N_{p t}} \omega_{p t}^{e} J_{p t}^{e} \sigma_{i j}^{e, p t},
$$

where $N_{\text {ele }}$ is the number of elements, $N_{p t}$ is the number of integration points on each element, and where $\omega, J$ are the 
weight and Jacobian on each integration point. An alternative is to use the surface integration (6), leading to

$$
\bar{\sigma}_{i j}=\frac{1}{V} \oint_{\partial V} t_{i} x_{j} d S=\frac{1}{V} \oint_{S} x_{j} d F_{i}=\frac{1}{V} \sum_{n=1}^{N_{\text {node }}} F_{i} x_{j}
$$

where $\boldsymbol{F}$ is the reaction force, and where $N_{\text {node }}$ is the number of nodes on the RVE boundary.

In case of linear material behavior, the effective tangent moduli components can easily be obtained by applying suitable macro-strain tensors $\bar{\varepsilon}$. For example, for

$$
\overline{\boldsymbol{\varepsilon}}=\left[\begin{array}{lll}
1 & 0 & 0 \\
0 & 0 & 0 \\
0 & 0 & 0
\end{array}\right],
$$

the elastic strain energy computed on the RVE yields

$$
U=\frac{V}{2} C_{1111}\left(\bar{\varepsilon}_{11}\right)^{2}=\frac{V}{2} C_{1111},
$$

from which $C_{1111}$ is inferred. The calculations for other components are straightforward.

\section{Periodic boundary condition enforcement by polynomial interpolation}

For periodic meshes, the existence of matching nodes on opposite faces of the RVE boundary allows to impose directly the periodic boundary condition. However, for arbitrary meshes, a new approach should be considered. In this work it is proposed to develop a method based on the polynomial interpolation. The idea of this method is that the displacement field on the RVE boundary can be interpolated by appropriate functions $\mathbb{S}$ whose forms are known, and such that (19) is satisfied. Because of its simplicity, a polynomial form is used as interpolation. In general, it is possible to express the general form of an interpolation by

$$
\boldsymbol{u}(s)=\mathbb{S}(\boldsymbol{s})=\sum_{i=0}^{n} \mathbb{N}_{i}(s) \boldsymbol{a}_{i},
$$

where each function $\mathbb{N}_{i}(\boldsymbol{s})$, with $i=0, . ., n$, only depends on the spatial variable $\boldsymbol{s}$, and where each variable $\boldsymbol{a}_{i}$, $i=0, . ., n$ is an independent first order tensor of degrees of freedom introduced in the finite element calculation.

In this work, the negative part of the RVE boundary is interpolated with the polynomial form $\mathbb{S}(s)$. As for the periodic boundary condition, equation (19) should be satisfied, and considering definition (14), the displacement field on the positive part should also be expressed in terms of $\mathbb{S}(s)$, leading to

$$
\begin{aligned}
& \boldsymbol{u}_{-}(s)=\mathbb{S}(s), \text { and } \\
& \boldsymbol{u}_{+}(s)=\mathbb{S}(s)+\bar{\varepsilon}\left(x^{+}-x^{-}\right),
\end{aligned}
$$

where the term $\overline{\boldsymbol{\varepsilon}}\left(\boldsymbol{x}^{+}-\boldsymbol{x}^{-}\right)$depends on the macroscopic strain and the RVE dimension. The polynomial interpolation for a 2-dimensional boundary value problem is illustrated in Fig. 4.

From (28)-(29), all degrees of freedom on the RVE boundary are expressed via the independent variables $\boldsymbol{a}_{i}$, which can now be specified. These independent variables can be selected as displacement of boundary mesh nodes or, more generally, as the new degrees of freedom of the system. In this work, the method is performed with two kinds of unidirectional interpolations: the global interpolation based on a Lagrange interpolation formulation, and the segmentation interpolation based on a cubic spline formulation. For 2-dimensional problems, these formulations are directly used to interpolate the displacement field on the boundary edges resulting in an edge polynomial interpolation formulation. For 3-dimensional problems, many surface interpolation formulations can be used, such as B-splines, to interpolate the displacement field on the boundary surfaces, leading to a surface polynomial interpolation formulation. In this work, in order to illustrate the efficiency of the method in the 3-dimensional case, a simple bi-linear patch Coons formulation is considered, but more evolved formulations can directly be derived. 


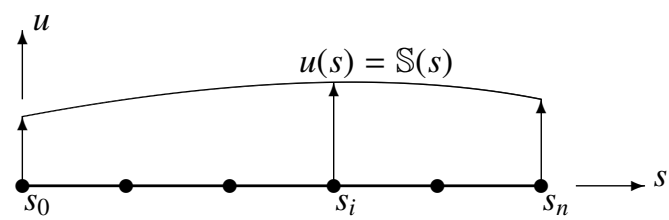

(a)

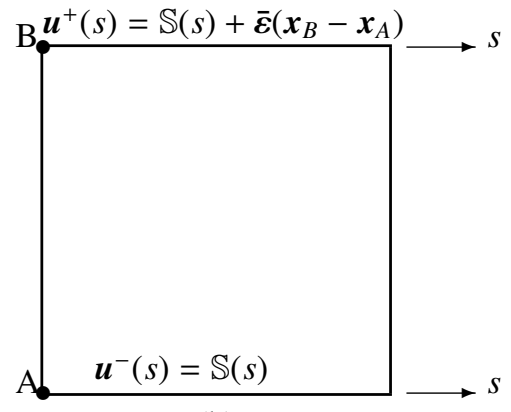

(b)

Figure 4: (a) Line polynomial interpolation $\mathbb{S}(s)$ and (b) Periodic boundary condition enforcement.

These edge and surface polynomial interpolation formulations are now presented. For simplicity, only the negative part of the RVE boundary (28) is considered, the positive part (29) being straightforwardly deduced.

\subsection{Edge polynomial interpolation}

As previously said, two formulations are suggested to interpolate the displacement field on an edge, the Lagrange formulation and the cubic spline formulation.

\subsubsection{Lagrange formulation}

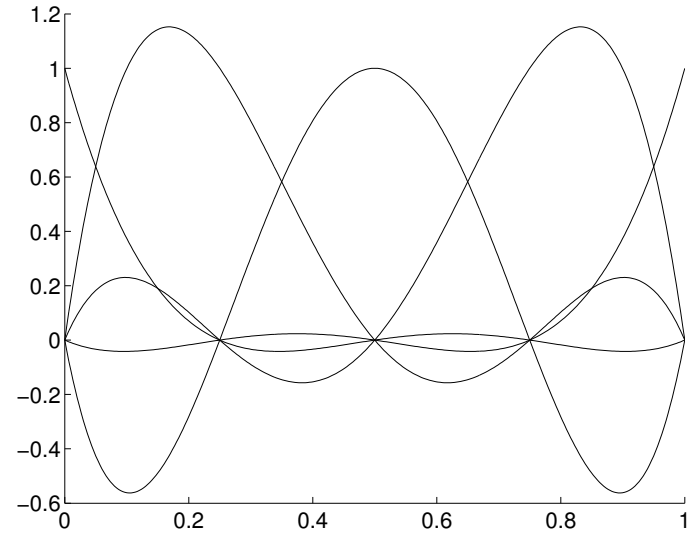

(a)

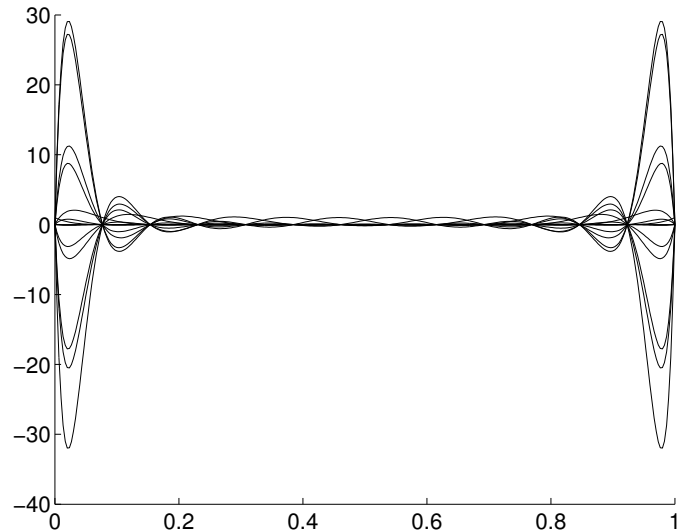

(b)

Figure 5: Lagrange interpolation functions $l_{i}$ for (a) Order 4 (b) Order 13

The displacement field on an edge, see Fig. 4a, is approximated by the polynomial $\mathbb{S}$ of order $n$,

$$
\mathbb{S}(s)=\sum_{i=0}^{n} \boldsymbol{a}_{i} s^{i}
$$

This polynomial form requests $n+1$ independent variables $\boldsymbol{a}_{i}$, with $i=0, . ., n$, defined from $n+1$ sampling displacements $\boldsymbol{u}_{0}, \ldots, \boldsymbol{u}_{n}$ evaluated at the sampling points $s_{0}, \ldots, s_{n}$. These sampling points can coincide to the mesh nodes or 
not. The polynomial $\mathbb{S}$ can thus be reformulated as

$$
\boldsymbol{u}=\mathbb{S}(s)=\sum_{i=0}^{n} l_{i}(s) \boldsymbol{u}_{i},
$$

where $l_{i}$ is the Lagrange polynomial associated to the sampling couple $\left(s_{i}, \boldsymbol{u}_{i}\right)$, and given by

$$
l_{i}(s)=\prod_{j=0, j \neq i}^{n} \frac{s-s_{j}}{s_{i}-s_{j}} .
$$

The function $l_{i}$ satisfies $l_{i}\left(s_{j}\right)=\delta_{i j}$ and the norm

$$
\sum_{i=0}^{n} l_{i}(s)=1 .
$$

Fig. 5 shows the polynomials $l_{i}(s)$, for the order 4 and 13, when the sampling nodes are regularly taken in the interval $[0,1]$. For the order 13 , there are some overshoots which can lead to instability in the results. So, the Lagrange interpolation is appropriate for the problem with few interpolation points only. Particularly, if $n=1$, the linear displacement boundary condition is recovered.

In the matrix form, the equation (31) is rewritten as

$$
\boldsymbol{u}(s)=\tilde{\mathbb{N}}(s) \tilde{\boldsymbol{q}}
$$

where $\tilde{\boldsymbol{q}}^{T}=\left[\boldsymbol{u}_{0}^{T} \ldots \boldsymbol{u}_{n}^{T}\right]$ is the independent variables vector and where $\tilde{\mathbb{N}}$ is the interpolation shape functions matrix. Finite implementation of this formulation is described in the next section.

\subsubsection{Cubic spline formulation}

For this formulation, an edge is divided into $N$ segments $\left[\left(s_{i-1}, \boldsymbol{u}_{i-1}\right) \quad\left(s_{i}, \boldsymbol{u}_{i}\right)\right]$ defined from the $n+1$ sampling couples $\left(s_{0}, \boldsymbol{u}_{0}\right), \ldots,\left(s_{N}, \boldsymbol{u}_{N}\right)$, see Fig. 4 a. At the extremities of each segment, two slope vectors $\boldsymbol{\theta}_{i-1}, \boldsymbol{\theta}_{i}$ are also defined, so the displacement field in each segment can be interpolated using the Hermite polynomials of order 3 :

$$
\begin{aligned}
& H_{1}(\xi)=1-3 \xi^{2}+2 \xi^{3} \\
& H_{2}(\xi)=d\left(\xi-2 \xi^{2}+\xi^{3}\right) \\
& H_{3}(\xi)=3 \xi^{2}-2 \xi^{3} \\
& H_{4}(\xi)=d\left(-\xi^{2}+\xi^{3}\right)
\end{aligned}
$$

where $\xi(s)=\frac{s-s_{i-1}}{s_{i}-s_{i-1}}$ and where $d=s_{i}-s_{i-1}$ is the segment length. Therefore, the displacement field can be interpolated on this segment from

$$
\boldsymbol{u}(s)=H_{1}(\xi(s)) \boldsymbol{u}_{i-1}+H_{2}(\xi(s)) \boldsymbol{\theta}_{i-1}+H_{3}(\xi(s)) \boldsymbol{u}_{i}+H_{4}(\xi(s)) \boldsymbol{\theta}_{i}
$$

Equation (39) can be rewritten in the matrix form:

$$
\boldsymbol{u}(s)=\tilde{\mathbb{N}}(\xi) \tilde{\boldsymbol{q}}
$$

where $\tilde{\boldsymbol{q}}^{T}=\left[\boldsymbol{u}_{0}^{T} \boldsymbol{\theta}_{0}^{T} \ldots \boldsymbol{u}_{N}^{T} \boldsymbol{\theta}_{N}^{T}\right]$ is the independent variables vector and $\tilde{\mathbb{N}}$ is the interpolation shape functions matrix.

\subsection{Surface polynomial interpolation}

For 3-dimensional problems, periodic boundary conditions can be enforced using the surface polynomial interpolation formulation. Toward this end, a unit surface problem in the axis system $(\xi, \eta)$ is considered, see Fig. 6, with the surface boundary $\Gamma=\Gamma_{A B} \cup \Gamma_{B C} \cup \Gamma_{C D} \cup \Gamma_{A D}$. On each part $\Gamma_{A B}, \Gamma_{A D}, \Gamma_{B C}, \Gamma_{C D}$ of the boundary, the displacement 


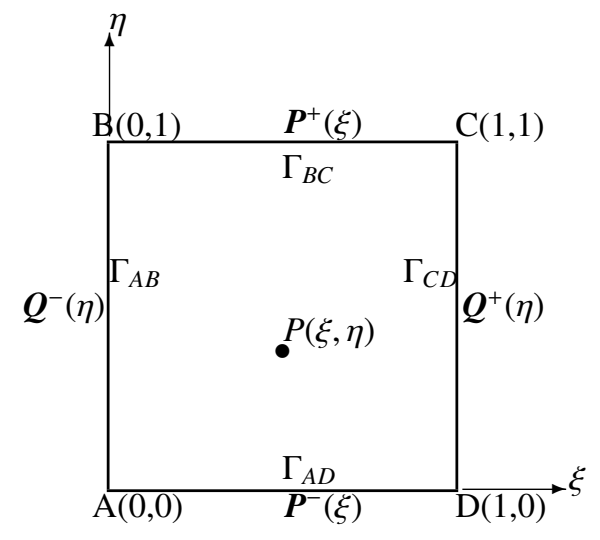

Figure 6: Surface polynomial interpolation, with surface boundary $\Gamma=\Gamma_{A B} \cup \Gamma_{B C} \cup \Gamma_{C D} \cup \Gamma_{A D}$.

field is interpolated using the Lagrange or cubic spline formulation presented in the previous sections, leading to the four interpolations $\boldsymbol{Q}^{-}(\eta), \boldsymbol{P}^{-}(\xi), \boldsymbol{Q}^{+}(\eta)$ and $\boldsymbol{P}^{+}(\xi)$, respectively.

As the final aim is to interpolate the displacement of an arbitrary point $P(\xi, \eta)$ on the surface, a Coons patch form $\mathbb{S}_{b}(\xi, \eta)$ is built from the interpolation functions of the surface boundary as

$$
\mathbb{S}_{b}(\xi, \eta)=\boldsymbol{S}_{b}^{1}(\xi, \eta)+\boldsymbol{S}_{b}^{2}(\xi, \eta)-\boldsymbol{S}_{b}^{3}(\xi, \eta)
$$

where

$$
\begin{aligned}
& \boldsymbol{S}_{b}^{1}(\xi, \eta)=(1-\eta) \boldsymbol{P}^{-}(\xi)+\eta \boldsymbol{P}^{+}(\xi), \\
& \boldsymbol{S}_{b}^{2}(\xi, \eta)=(1-\xi) \boldsymbol{Q}^{-}(\eta)+\xi \boldsymbol{Q}^{+}(\eta), \text { and } \\
& \boldsymbol{S}_{b}^{3}(\xi, \eta)=(1-\xi)(1-\eta) \boldsymbol{u}_{A}+(1-\xi) \eta \boldsymbol{u}_{B}+\xi \eta \boldsymbol{u}_{C}+\xi(1-\eta) \boldsymbol{u}_{D}
\end{aligned}
$$

Thus, the displacement of an arbitrary node $P(\xi, \eta)$ on the surface is expressed from $\mathbb{S}_{b}$ :

$$
\boldsymbol{u}(\xi, \eta)=\mathbb{S}_{b}(\xi, \eta) .
$$

As the periodic boundary condition is considered, all edges of the surface boundary must also satisfy this condition, yielding

$$
\begin{aligned}
& \boldsymbol{P}^{+}(\xi)=\boldsymbol{P}^{-}(\xi)+\overline{\boldsymbol{\varepsilon}}\left(\boldsymbol{x}_{B}-\boldsymbol{x}_{A}\right), \text { and } \\
& \boldsymbol{Q}^{+}(\eta)=\boldsymbol{Q}^{-}(\eta)+\overline{\boldsymbol{\varepsilon}}\left(\boldsymbol{x}_{D}-\boldsymbol{x}_{A}\right) .
\end{aligned}
$$

For $\xi$ or $\eta$ equal to one, these two conditions imply

$$
\begin{aligned}
& \boldsymbol{u}_{B}=\boldsymbol{u}_{A}+\overline{\boldsymbol{\varepsilon}}\left(\boldsymbol{x}_{B}-\boldsymbol{x}_{A}\right), \\
& \boldsymbol{u}_{C}=\boldsymbol{u}_{A}+\overline{\boldsymbol{\varepsilon}}\left(\boldsymbol{x}_{C}-\boldsymbol{x}_{A}\right), \text { and } \\
& \boldsymbol{u}_{D}=\boldsymbol{u}_{A}+\overline{\boldsymbol{\varepsilon}}\left(\boldsymbol{x}_{D}-\boldsymbol{x}_{A}\right) .
\end{aligned}
$$

Finally, substituting Eqs. (46-50) in the interpolation (41) leads to

$$
\mathbb{S}_{b}(\xi, \eta)=\boldsymbol{P}^{-}(\xi)+\boldsymbol{Q}^{-}(\eta)-\boldsymbol{u}_{A},
$$

and the displacement of the arbitrary point $P(\xi, \eta)$ on the surface is expressed by:

$$
\boldsymbol{u}(\xi, \eta)=\boldsymbol{P}^{-}(\xi)+\boldsymbol{Q}^{-}(\eta)-\boldsymbol{u}_{\boldsymbol{A}}
$$


In the matrix form, this last relation is rewritten

$$
\boldsymbol{u}(\xi, \eta)=\tilde{\mathbb{N}}_{P}(\xi) \tilde{\boldsymbol{q}}_{P}+\tilde{\mathbb{N}}_{Q}(\eta) \tilde{\boldsymbol{q}}_{Q}=\tilde{\mathbb{N}}(\xi, \eta) \tilde{\boldsymbol{q}}
$$

where $\tilde{\boldsymbol{q}}$ is the independent variables vector, and where $\tilde{\mathbb{N}}$ is the interpolation shape functions matrix.

\section{Finite element implementation}

In this section the implementation of the proposed formulations to enforce periodic boundary condition is presented. Moreover, as the solutions will be compared with the matching node formulation traditionally used for periodic meshes, that method is also recalled.

\subsection{Classical implementations for periodic mesh}

In order to impose the periodic boundary condition, the RVE boundary must be discretized using "the mapping nodes formulation". Each mapping node includes a node on negative boundary $\partial V^{-}$and a node on positive boundary $\partial V^{-}$, see Fig. 3. Suppose that $p$ mapping nodes are created from two opposite sides, the periodic boundary condition (19) is directly written as

$$
\boldsymbol{u}_{i}^{+}-\boldsymbol{u}_{i}^{-}=\overline{\boldsymbol{\varepsilon}}\left(\boldsymbol{x}_{i}^{+}-\boldsymbol{x}_{i}^{-}\right) \quad i=1, \ldots, p
$$

The solution of resulting boundary value problem is found by minimizing the energy function

$$
\mathbb{E}=\frac{1}{2} \boldsymbol{u}^{T} \boldsymbol{K} \boldsymbol{u}-\boldsymbol{u}^{T} \boldsymbol{F}
$$

where $\boldsymbol{u}$ is the displacement vector, $\boldsymbol{K}$ is the stiffness matrix, and $\boldsymbol{F}$ is the external loading vector, subject to the periodic constraint built from (54).

In order to enforce the equations (54) in the finite element context, two methods are generally used: the Lagrange multiplier method and the constraint elimination method. The Lagrange multiplier method is detailed in [2] and the constraint elimination method is briefly recalled.

\subsubsection{Constraint elimination method}

A common method to enforce the equation (54) is the constraint elimination method, which is based on the substitution of the node displacements on the positive boundary by those on the negative boundary of the RVE:

$$
\boldsymbol{u}_{i}^{+}=\boldsymbol{u}_{i}^{-}+\bar{\varepsilon}\left(\boldsymbol{x}_{i}^{+}-\boldsymbol{x}_{i}^{-}\right), \quad i=1, \ldots, p .
$$

The stationary point of the functional $\mathbb{E}(55)$, without constraints, reads

$$
\delta \boldsymbol{u}^{T}(\boldsymbol{K} \boldsymbol{u}-\boldsymbol{F})=0 .
$$

The displacements vector can be reorganized into 3 parts: internal displacements vector $\boldsymbol{u}_{\boldsymbol{i}}$, positive node displacements vector $\boldsymbol{u}_{+}$and negative node displacements vector $\boldsymbol{u}_{-}$:

$$
\boldsymbol{u}=\left[\begin{array}{c}
\boldsymbol{u}_{i} \\
\boldsymbol{u}_{+} \\
\boldsymbol{u}_{-}
\end{array}\right]
$$

and equation (56) can be restated as

$$
\boldsymbol{u}_{+}=\boldsymbol{u}_{-}+\boldsymbol{g}
$$


where $\boldsymbol{g}$ is calculated from the macroscopic strain tensor $\overline{\boldsymbol{\varepsilon}}$. Accordingly, the stiffness matrix $\boldsymbol{K}$ and external force vector $\boldsymbol{F}$ of equation (57) can be reorganized in the form

$$
\left[\begin{array}{lll}
\delta \boldsymbol{u}_{i}^{T} & \delta \boldsymbol{u}_{+}^{T} & \delta \boldsymbol{u}_{-}^{T}
\end{array}\right]\left(\left[\begin{array}{ccc}
\boldsymbol{K}_{i i} & \boldsymbol{K}_{i+} & \boldsymbol{K}_{i-} \\
\boldsymbol{K}_{+i} & \boldsymbol{K}_{++} & \boldsymbol{K}_{+-} \\
\boldsymbol{K}_{-i} & \boldsymbol{K}_{-+} & \boldsymbol{K}_{--}
\end{array}\right]\left[\begin{array}{c}
\boldsymbol{u}_{i} \\
\boldsymbol{u}_{+} \\
\boldsymbol{u}_{-}
\end{array}\right]-\left[\begin{array}{c}
\boldsymbol{F}_{i} \\
\boldsymbol{F}_{+} \\
\boldsymbol{F}_{-}
\end{array}\right]\right)=0 .
$$

Introducing equation (59) in this expression, terms $\boldsymbol{u}_{+}$are substituted by $\boldsymbol{u}_{-}$, yielding

$$
\left[\begin{array}{ll}
\delta \boldsymbol{u}_{i}^{T} & \delta \boldsymbol{u}_{-}^{T}
\end{array}\right]\left(\left[\begin{array}{cc}
\boldsymbol{K}_{i i} & \boldsymbol{K}_{i+}+\boldsymbol{K}_{i-} \\
\boldsymbol{K}_{+i}+\boldsymbol{K}_{-i} & \boldsymbol{K}_{++}+\boldsymbol{K}_{+-}+\boldsymbol{K}_{-+}+\boldsymbol{K}_{--}
\end{array}\right]\left[\begin{array}{c}
\boldsymbol{u}_{i} \\
\boldsymbol{u}_{-}
\end{array}\right]-\left[\begin{array}{c}
\boldsymbol{F}_{i}-\boldsymbol{K}_{i+} \boldsymbol{g} \\
\boldsymbol{F}_{+}+\boldsymbol{F}_{-}-\left(\boldsymbol{K}_{++}+\boldsymbol{K}_{-+}\right) \boldsymbol{g}
\end{array}\right]\right)=0,
$$

or again

$$
\delta \tilde{\boldsymbol{u}}^{T}(\tilde{\boldsymbol{K}} \tilde{\boldsymbol{u}}-\tilde{\boldsymbol{F}})=0
$$

where $\tilde{\boldsymbol{u}}^{T}=\left[\begin{array}{ll}\boldsymbol{u}_{i}^{T} & \boldsymbol{u}_{-}^{T}\end{array}\right]$. As $\delta \tilde{\boldsymbol{u}}^{T}$ is arbitrary and kinematically admissible, the resulting linear systems of algebraic equations of unknowns $\tilde{\boldsymbol{u}}$ reads

$$
\tilde{\boldsymbol{K}} \tilde{\boldsymbol{u}}-\tilde{\boldsymbol{F}}=0
$$

This method allows reducing the number of unknowns of the system.

\subsection{Polynomial interpolation implementation}

The two methods presented above are only suitable for periodic meshes where the mapping node condition is always guaranteed. For a general mesh, this condition is no longer satisfied, and the methods presented in section 3 , based on the polynomial interpolation, can be used after implementation in the finite element framework. The polynomial interpolation formulation gives a polynomial form of the nodal displacements on negative part of the RVE boundary, from Eq. (34) for a Lagrange form, from Eq. (40) for a cubic spline form, or from (53) for the 3D cases. For all these cases, the interpolation form is summarized by

$$
\boldsymbol{u}_{-}=\tilde{\mathbb{N}} \tilde{\boldsymbol{q}} .
$$

Applying the periodic boundary condition (19) yields the nodal displacement interpolation on the positive edge:

$$
\boldsymbol{u}_{+}=\tilde{\mathbb{N}} \tilde{\boldsymbol{q}}+\overline{\boldsymbol{\varepsilon}}\left(\boldsymbol{x}^{+}-\boldsymbol{x}^{-}\right)
$$

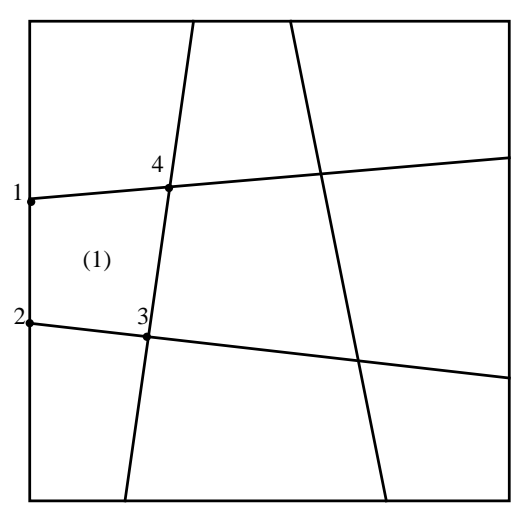

Figure 7: Simple mesh for illustration purpose. 
To illustrate the implementation of the polynomial interpolation, the simple 2D-mesh of Fig. 7 is considered. The constraints will be enforced for element 1 defined from the 4 nodes $(1,2,3,4)$. In the finite element interpolation formulation, the displacement field inside this element reads

$$
\begin{aligned}
\boldsymbol{u}_{e}(x, y) & =N_{1}(x, y) \boldsymbol{u}_{1}+N_{2}(x, y) \boldsymbol{u}_{2}+N_{3}(x, y) \boldsymbol{u}_{3}+N_{4}(x, y) \boldsymbol{u}_{4}=\mathbb{N}_{e} \boldsymbol{q}_{e} \text {, with } \\
\boldsymbol{q}_{e}^{T} & =\left[\begin{array}{llll}
\boldsymbol{u}_{1}^{T} & \boldsymbol{u}_{2}^{T} & \boldsymbol{u}_{3}^{T} & \boldsymbol{u}_{4}^{T}
\end{array}\right] .
\end{aligned}
$$

As nodes 1 and 2 of this element lie on the boundary, assuming on the negative part, the polynomial interpolation implies

$$
\begin{aligned}
& \boldsymbol{u}_{1}=\mathbb{N}_{1} \tilde{\boldsymbol{q}}, \text { and } \\
& \boldsymbol{u}_{2}=\mathbb{N}_{2} \tilde{\boldsymbol{q}} .
\end{aligned}
$$

In case they lie on the positive part of the boundary, these last two relations become :

$$
\begin{aligned}
& \boldsymbol{u}_{1}=\mathbb{N}_{1} \tilde{\boldsymbol{q}}+\overline{\boldsymbol{\varepsilon}}\left(\boldsymbol{x}^{+}-\boldsymbol{x}^{-}\right), \text {and } \\
& \boldsymbol{u}_{2}=\mathbb{N}_{2} \tilde{\boldsymbol{q}}+\overline{\boldsymbol{\varepsilon}}\left(\boldsymbol{x}^{+}-\boldsymbol{x}^{-}\right) .
\end{aligned}
$$

Thus, the displacement field (67) of the element nodes can be restated as

$$
\boldsymbol{q}_{e}=\left[\begin{array}{l}
\boldsymbol{u}_{1} \\
\boldsymbol{u}_{2} \\
\boldsymbol{u}_{3} \\
\boldsymbol{u}_{4}
\end{array}\right]=\left[\begin{array}{c}
\mathbb{N}_{1} \tilde{\boldsymbol{q}}+\langle\boldsymbol{g}\rangle \\
\mathbb{N}_{2} \tilde{\boldsymbol{q}}+\langle\boldsymbol{g}\rangle \\
\boldsymbol{u}_{3} \\
\boldsymbol{u}_{4}
\end{array}\right]=\mathbb{L}_{e} \tilde{\boldsymbol{q}}_{e}+\tilde{\boldsymbol{g}}_{e},
$$

where $\tilde{\boldsymbol{q}}_{e}^{T}=\left[\begin{array}{lll}\boldsymbol{u}_{3}^{T} & \boldsymbol{u}_{4}^{T} & \tilde{\boldsymbol{q}}^{T}\end{array}\right]$ is the new elementary vector of degrees of freedom, where $\mathbb{L}_{e}$ is the mapping matrix which allows transforming the element displacement vector $\boldsymbol{q}_{e}$ into the constraint displacement vector $\tilde{\boldsymbol{q}}_{e}$, where $\langle\boldsymbol{g}\rangle$ is the contribution of $\overline{\boldsymbol{\varepsilon}}\left(\boldsymbol{x}^{+}-\boldsymbol{x}^{-}\right)$for nodes lying on the positive boundary part and is equal to zero for nodes lying on the negative boundary part, and where $\tilde{\boldsymbol{g}}_{e}$ is the elementary vector related to $\langle\boldsymbol{g}\rangle$.

The stationary point of the functional $\mathbb{E}(55)$, without constraints, written in terms of the elementary values, reads

$$
\sum_{e}\left(\delta \boldsymbol{q}_{e}^{T} \boldsymbol{K}_{e} \boldsymbol{q}_{e}\right)-\sum_{e}\left(\delta \boldsymbol{q}_{e}^{T} \boldsymbol{F}_{e}\right)=0
$$

where $\delta \boldsymbol{q}_{e}$ is the arbitrary virtual displacement, where $\boldsymbol{K}_{e}$ is the elementary stiffness matrix and where $\boldsymbol{F}_{e}$ is the elementary force vector. According to Eq. (72), the virtual displacement becomes:

$$
\delta \boldsymbol{q}_{e}=\mathbb{L}_{e} \boldsymbol{\delta} \tilde{\boldsymbol{q}}_{e},
$$

and substituting this relation into (73), the new finite element formulation of non-constrained displacement $\tilde{q}_{e}$ reads

$$
\sum_{e}\left(\delta \tilde{\boldsymbol{q}}_{e}^{T} \mathbb{L}_{e}^{T} \boldsymbol{K}_{e} \mathbb{L}_{e} \tilde{\boldsymbol{q}}_{e}\right)-\sum_{e}\left(\delta \tilde{\boldsymbol{q}}_{e}^{T} \mathbb{L}_{e}^{T} \boldsymbol{F}_{e}-\delta \tilde{\boldsymbol{q}}_{e}^{T} \mathbb{L}_{e}^{T} \boldsymbol{K}_{e} \tilde{\boldsymbol{g}}_{e}\right)=0
$$

or

$$
\sum_{e} \delta \tilde{\boldsymbol{q}}_{e}^{T}\left(\tilde{\boldsymbol{K}}_{e} \tilde{\boldsymbol{q}}_{e}-\tilde{\boldsymbol{F}}_{e}\right)=0
$$

where $\tilde{\boldsymbol{K}}_{e}=\mathbb{L}_{e}^{T} \boldsymbol{K}_{e} \mathbb{L}_{e}$ is the modified elementary stiffness matrix and where $\tilde{\boldsymbol{F}}_{e}=\mathbb{L}_{e}^{T}\left(\boldsymbol{F}_{e}-\boldsymbol{K}_{e} \tilde{\boldsymbol{g}}_{e}\right)$ is the modified elementary force vector.

Equation (76) defines the implementation of the polynomial interpolation. In theory, the independent variables $\tilde{\boldsymbol{q}}$ can be chosen independently of the existing nodal displacements on the RVE boundary. Implementation of the method is conducted in the Gmsh framework [17]. 


\section{Polynomial interpolation method analysis at constant RVE size}

To demonstrate the ability of the described interpolation method to enforce periodic boundary conditions on arbitrary meshes, as first step, the effect of the users parameters is studied when extracting the effective material properties of a constant-size RVE made of an heterogeneous material. In this section a 2D-RVE is considered, and the methods considered are successively the polynomial interpolations based on the Lagrange and on the cubic spline interpolations, whose parameters are respectively the polynomial order and the number of segments.

\subsection{Numerical models}

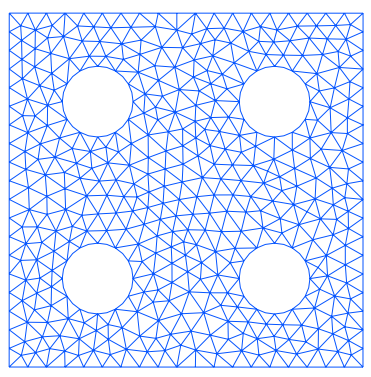

(a)

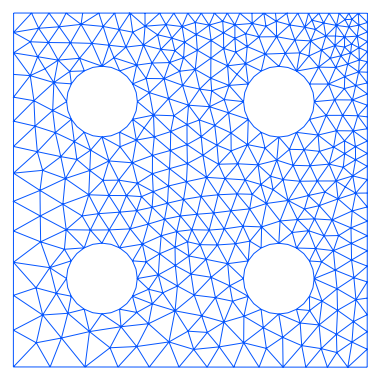

(b)

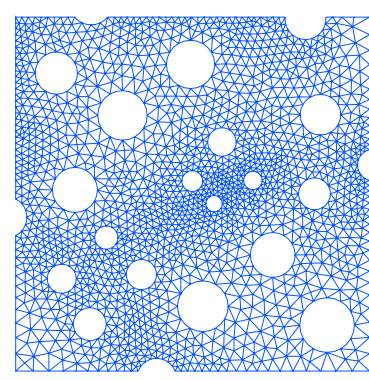

(c)

Figure 8: RVEs used in the analysis: (a) Periodic mesh, (b) Non-periodic mesh generated from periodic hole distribution, $\left(\right.$ RVE size $L=2 L_{\text {ref }}$, void fraction 0.1257 ) and (c) Non- periodic mesh generated from a random hole distribution, (RVE size $L=3 L_{\text {ref }}$, void fraction 0.1657 ).

The polynomial interpolation algorithm is used to impose the periodic boundary condition on a 2D-RVE. As stated previously, this method can be used for two kinds of mesh models:

- One is the periodic mesh where the mapping node condition is satisfied. For this mesh, a square RVE with 4 identical holes regularly arranged, dimension $2 L_{\mathrm{ref}}\left(L_{\mathrm{ref}}\right.$ is the size of a cell with one hole) of void fraction 0.1257 is considered, see Fig. 8a.

- The other one is the non-periodic mesh where the mapping node condition is not satisfied. In this case, two RVEs are considered: (i) a square RVE with 4 identical holes regularly arranged, dimension $2 L_{\text {ref }}$, see Fig. $8 \mathrm{~b}$, and (ii) a square RVE with holes randomly distributed, dimension $3 L_{\mathrm{ref}}\left(L_{\mathrm{ref}}\right.$ is the reference cell size, see definition in Appendix A) of void fraction 0.1657, see Fig. 8c. ${ }^{2}$

The material used in all models is elastic (Young modulus $E=70 \mathrm{GPa}$ and Poisson ration $v=0.3$, leading to $G_{\mathrm{ref}}=26.92$ $\mathrm{GPa})$, and the calculation is investigated in the plane strain state $\left(\varepsilon_{z z}=\varepsilon_{x z}=\varepsilon_{y z}=0\right)$. Quadratic iso-parametric triangular elements are used in order to better represent the displacement field and the circular holes.

In this study, the equivalent in-plane shear modulus is used to analyze the sensibility of the parameters. To calculate this effective modulus, a pure shearing state is applied to the RVE with the macro-strain tensor:

$$
\bar{\varepsilon}=\left[\begin{array}{cc}
0 & 0.5 \\
0.5 & 0
\end{array}\right]
$$

The resulting macro-stress tensor is computed by using successively the direct volume averaging procedure (23) or the surface averaging procedure (24). The resulting effective shear stress represents the effective shear modulus $G$.

\footnotetext{
${ }^{2}$ Note that when voids intersect with opposite sides of the RVE boundary, the traction free boundary condition is not strongly imposed as the voids do not intersect in a periodical way. This enforcement is weakly satisfied through the periodic boundary conditions. Although this is not rigorous, the error is of the same order as considering a RVE of limited size to extract the properties
} 
The reference computation is obtained from the constraint elimination method presented in section 4.1.1, which demands the periodic mesh of Fig. 8a. The computed macro-stress tensor is

$$
\bar{\sigma}=\left[\begin{array}{cc}
0.0011 & 18.0168 \\
18.0168 & 0.0009
\end{array}\right] \mathrm{GPa} .
$$

Let us remark the presence of small diagonal values resulting from the finite element discretization error, which are negligible in comparison to the out of diagonal values.

The effective shear modulus $G$ for the $2 \mathrm{D}-\mathrm{RVE}$, of void-fraction $0.1257 \mathrm{is} 18.0168 \mathrm{GPa}$, or $G / G_{\text {ref }}=0.669$. This reference value is the reference solution for a given FE discretization and for a periodic material of void-fraction 0.1257. Indeed, as the structure is periodic, and as the periodic boundary condition is rigorously enforced, the extracted effective shear modulus is the one of the heterogeneous material, the only error resulting from the finite-element discretization. Thus we can compare the results obtained with the new interpolation method, in terms of the of the number of degrees of freedom of the boundary displacement field, to this reference.

For the 2D-RVE, the number of degrees of freedom which is necessary to interpolate the displacement field on the RVE sides can be deduced either from the polynomial order $n$ of the Lagrange interpolation or from the number of segments $N$ of the cubic spline interpolation, as

$$
\begin{aligned}
N_{\text {dof }}^{\text {Lagrange }} & =6+4(n-1), \\
N_{\text {dof }}^{\text {spline }} & =12+8(N-1) .
\end{aligned}
$$

In what follows, the influence of the polynomial interpolation degree (polynomial order and number of segments) on the computed effective properties of material is expressed in terms of the number of degrees of freedom used for the boundary displacement field.

\subsection{Results for periodic micro-structures}

The polynomial interpolation method is used to enforce the periodic boundary condition for both the periodic mesh, see Fig. 8a, and the non-periodic mesh, see Fig. 8b, of the periodic structure. The results obtained with the polynomial interpolations based on, successively, the Lagrange and the cubic spline interpolations, whose parameters are respectively the polynomial order and the number of segments, are reported on Figs. 9a, 10, and 11.

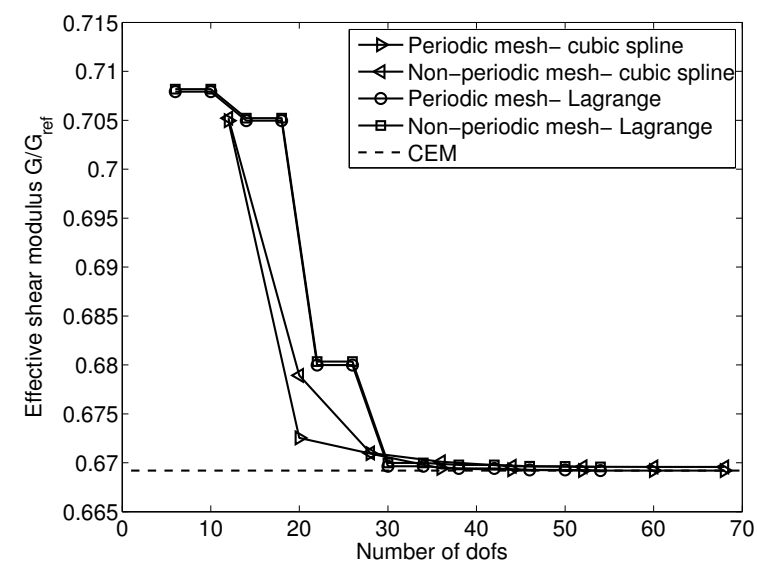

(a)

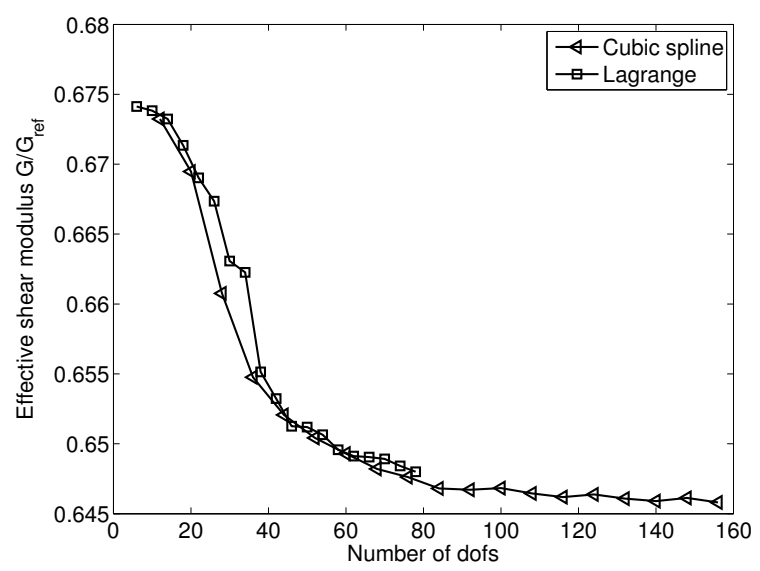

(b)

Figure 9: Influence of polynomial interpolation parameters on the in-plane effective shear modulus in terms of the number of degree of freedom of the boundary displacement field: (a) Periodic structure and (b) Non-periodic structure. Results with the periodic structures are compared to the result given by the constraint elimination method (CEM) computed with periodic mesh. 


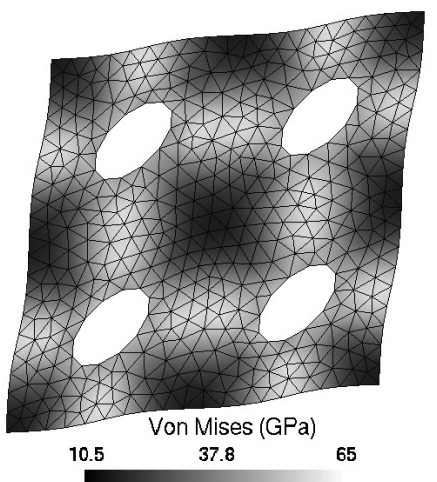

(a)

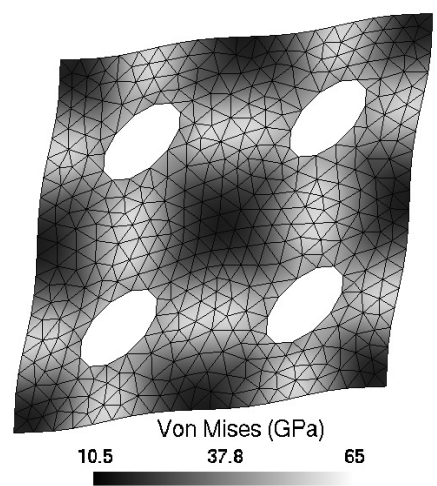

(b)

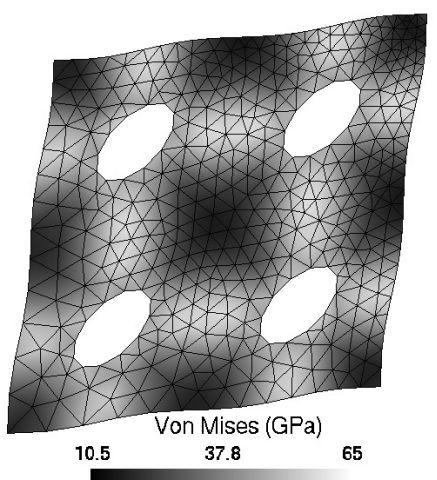

(c)

Figure 10: Distribution of von Mises stress in the periodic micro-structure, for the macro strain tensor (77), obtained with (a) The constraint elimination method (CEM), (b) The Lagrange interpolation (polynomial order is 7, periodic mesh) and (c) The Lagrange interpolation (polynomial order is 7 , non-periodic mesh).

\subsubsection{Influence of the polynomial order for the Lagrange interpolation}

The Lagrange interpolation method described in section 3.1.1 is characterized by the polynomial order $n$. The polynomial interpolation implementation is equivalent to introducing constraints in the system, with the two extreme cases: The periodic boundary condition is equivalent to the less constrained case (polynomial interpolation with infinity order) and the linear displacement boundary condition is equivalent to the most constrained case (polynomial interpolation with order 1). Thus, the results given by the Lagrange interpolation method must converge to the effective modulus value $G=18.0168 \mathrm{GPa}$ when the polynomial order increases.

Fig. 9a represents the effective in-plane shear modulus extracted for the structured material in function of the polynomial order, for both periodic and non-periodic meshes. This figure shows the convergence to the result obtained by the constraint elimination method.

For this RVE of size $L=2 L_{\text {ref }}$, the degree 7 (30 dofs) leads to a really accurate estimation (lower than $0.1 \%$ ). It means that this order is already sufficient to capture adequately the displacement field on RVE boundary. Of course, a larger RVE demands a larger order to interpolate more accurately as it will be seen in next section. We also observe that the results given by the odd orders and even orders are almost the same. This point can be explained by the symmetry of the periodic arrangement. So the displacement field on boundaries is interpolated by a polynomial with odd order (even coefficient being null). In all following, calculations with periodic structures are obtained with an odd interpolation order.

Finally, Fig. 10b and Fig. 10c present the deformations and the von Mises stress distributions of the RVE obtained with the polynomial order 7 for both periodic and non-periodic meshes respectively. These resulst are shown to be similar to the ones obtained with the constraint elimination method, Fig. 10a.

\subsubsection{Influence of the number of segments for the cubic spline interpolation}

The parameter of the cubic spline interpolation formulation, presented in section 3.1.2, is the number of segments $N$. This method is implemented for both periodic and non-periodic meshes. In theory, an arbitrary curve can be approximated by an infinite number of cubic segments. In others words, the results given by the cubic spline interpolation method must converge to the results obtained with the constraint elimination method when the number of segments increases. This convergence property is presented in Fig. 9a, where it can be seen that when the number of segments $N$ is larger than 4 (36 dofs), the results for both periodic and non-periodic meshes are very close to the results obtained with constraint elimination method. This means 4 segments are sufficient to interpolate the displacement on the RVE boundary. Of course, a larger RVE demands a larger number of segments.

Fig. 11b and Fig. 11c show the deformations and the von Mises stress distributions of the RVE with periodic and non-periodic meshes respectively, showing a good agreement with the results obtained by the constraint elimination 


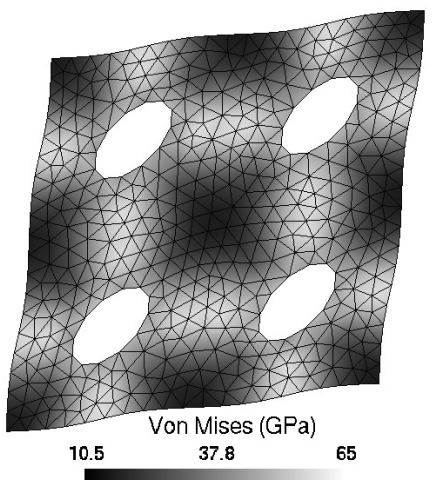

(a)

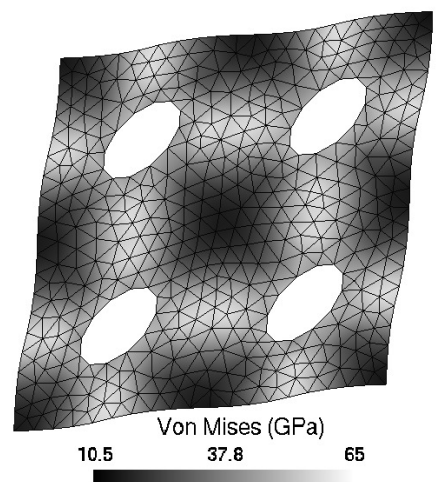

(b)

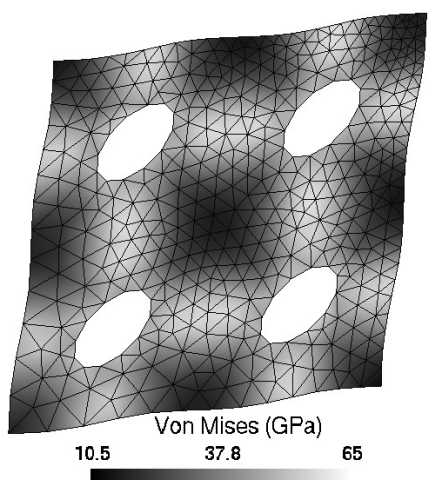

(c)

Figure 11: Distribution of Von-Mises stress in the periodic micro-structure, for the macro strain tensor (77), obtained with (a) The constraint elimination method (CEM), (b) The cubic spline interpolation (number of segment is 4, periodic mesh) and (c) The cubic spline interpolation (number of segment is 4 , non-periodic mesh).

method, Fig. 11a.

\subsection{Results for non-periodic micro-structures}

In this section, the RVE illustrated on Fig. 8c, and exhibiting a non-periodic micro-structure, is considered. This RVE consists of circular voids randomly distributed, its size is $3 L_{\text {ref }}$ and its void fraction is 0.1657 . For this nonperiodic structure, the mapping node condition required to enforce the periodic boundary condition by the constraint elimination method is not satisfied, and the polynomial interpolation method has to be considered.

The extracted in-plane effective shear modulus is reported on Fig. $9 \mathrm{~b}$ in terms of the necessary number of degrees of freedom of the boundary displacement field used. However, let us remark that for a non-periodic micro-structure the effective shear modulus should be extracted for a RVE size much larger than the void diameter. If this is not the case, the solution might depend on the configuration, meaning that two RVE with the same void fraction have different effective properties, as it will be illustrated in the next section. Nevertheless, keeping this in mind, a parameter study can still be performed on the considered RVE.

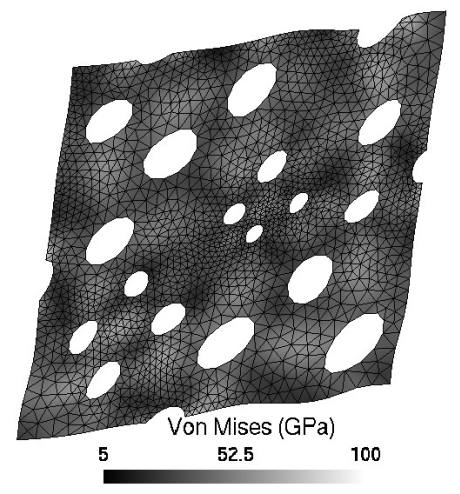

(a)

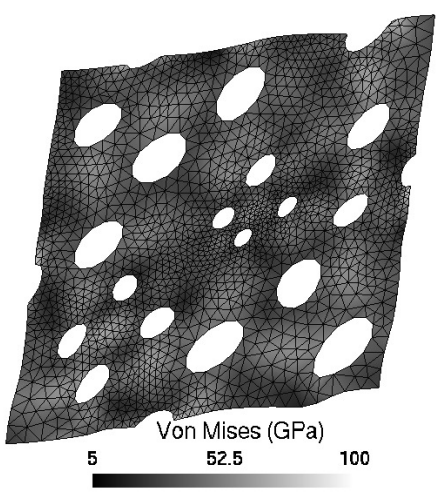

(b)

Figure 12: Distribution of von Mises stress of the non-periodic micro-structure obtained with (a) The Lagrange interpolation (polynomial order is 11) and (b) The cubic spline interpolation (number of cubic segments is 10). 


\subsubsection{Influence of the polynomial order for the Lagrange interpolation}

As for the periodic structure, a larger polynomial order corresponds to less constraints applied to the system, and thus the effective shear modulus should decrease, as it is confirmed by the numerical simulations on Fig. 9b. For this RVE, a polynomial order larger than 11 (46 dofs) leads to an acceptable estimation, and a much better approximation than the linear displacement boundary condition, which result is given by the polynomial order 1. A larger RVE demands a larger order.

\subsubsection{Influence of the number of segments for cubic spline interpolation}

As for the periodic structure, Fig. 9b confirms the convergence of the method when the number of segments increases. In this case, the number of segments 10 ( $84 \mathrm{dofs}$ ) is sufficient to interpolate the displacement field on the RVE boundary. A larger RVE demands a larger number of segments.

Fig. 12 shows the deformation and the Von-Mises stress distribution of the RVE with the two interpolation methods, which are in excellent agreement.

\section{Convergence study in terms of the RVE size}

For periodic-micro-structures, as on Fig 8a, it is always possible to find a reduced-size RVE, which, combined to the periodic boundary condition, leads to the correct evaluation of the effective properties, within numerical errors resulting from the finite element discretization. On the other hand, for non-periodic micro-structures, as on Fig 8c, the material properties depend on the micro-structure heterogeneity distribution and on the RVE size. When the RVE size increases, the extracted effective material properties converges toward the homogenized material properties. For lower RVE size, the extracted properties depend on the sample: two samples of the same material will not have exactly the same heterogeneity distribution, and thus will exhibit two different material properties. Note that when non-linear effects such as brittle fracture occurs, the representative nature of the volume is less straightforward to be defined [18], and the problem becomes more complex.

In this section these two behaviors are verified by applying the periodic boundary condition using the newly implemented polynomial interpolation. Results are also compared with the solutions obtained with the constraint elimination method, when available, and the linear displacement boundary condition. In particular it will be shown that the periodic boundary condition always lead to a better approximation of the effective material properties than the linear displacement boundary condition.

\subsection{Definition of the RVEs}

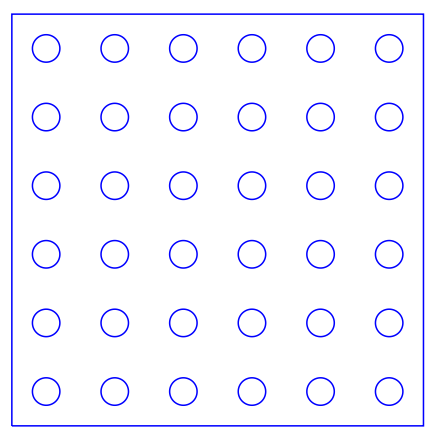

(a)

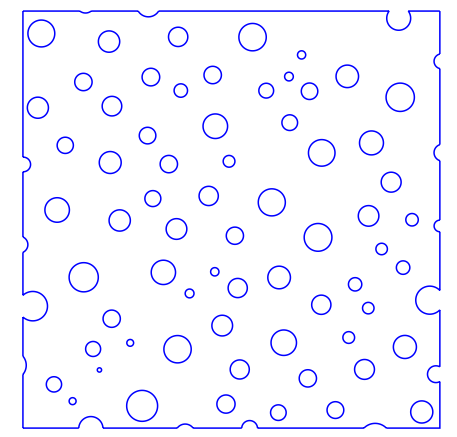

(b)

Figure 13: (a) Periodic structure and (b) Non-periodic structure.

For the periodic structures, the RVE is simply chosen by repeating a unit cell throughout the structure. This unit cell is a square with a single hole in the center, with a resulting void fraction of 0.1257 . As our aim is to study the polynomial interpolation method, periodic and non-periodic meshes are considered. An example of the periodic mesh 
and non-periodic mesh for the repeat unit cell with 4 holes is shown in Fig. 8a and b respectively. To illustrate the convergence of the method with the RVE size, this size ranges from $1 L_{\text {ref }}$ corresponding to 1 hole in the RVE center to $6 L_{\text {ref }}$ corresponding to 36 holes periodically arranged (Fig. 13a).

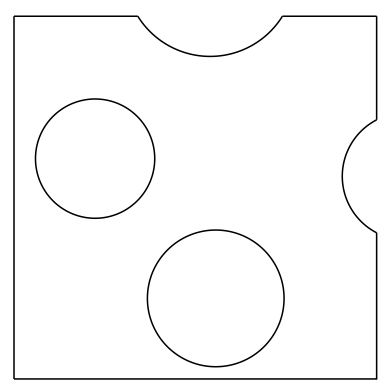

(a) $\frac{L}{L_{\text {ref }}}=1$

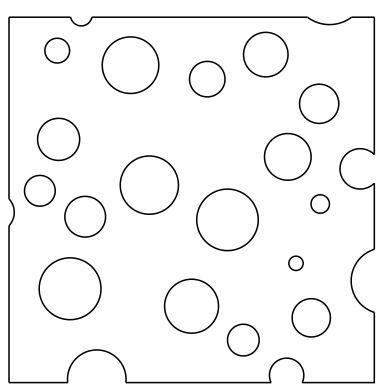

(b) $\frac{L}{L_{\mathrm{ref}}}=3$

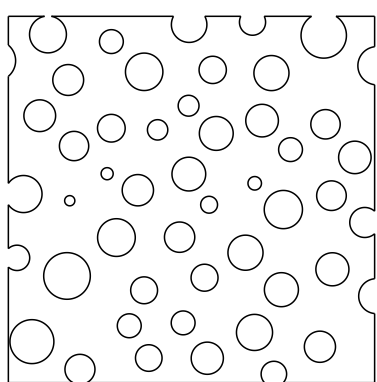

(c) $\frac{L}{L_{\mathrm{ref}}}=5$

Figure 14: Single realization of the randomly distributed hole material for different RVE sizes

The non-periodic structures, shown in Fig. 13b, are characterized by a random distribution of holes. Thus for a given RVE size, there is an infinite number of existing configurations, and thus of effective material properties. Therefore, for each RVE size, a large number of realizations should be considered in order to predict the macroscopic properties. For statistical reasons, a smaller RVE size demands a larger number of realizations, but a small RVE can still be used to extract the material properties if a sufficient number of realizations is provided [11, 13]. Figs. 14a to c show a typical single realizations of RVE sizes $1 L_{\mathrm{ref}}, 3 L_{\mathrm{ref}}$, and $5 L_{\mathrm{ref}}$ respectively. The resulting meshes are always non-periodic (Fig. 8c).

\subsection{Periodic structures}

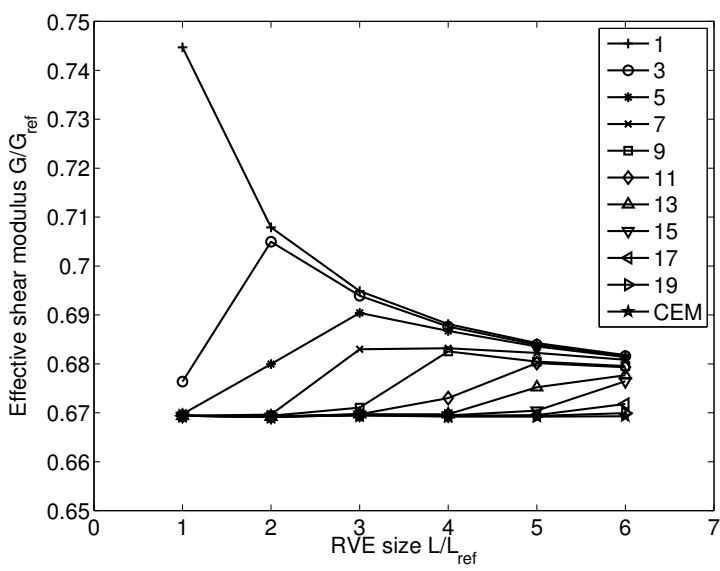

(a)

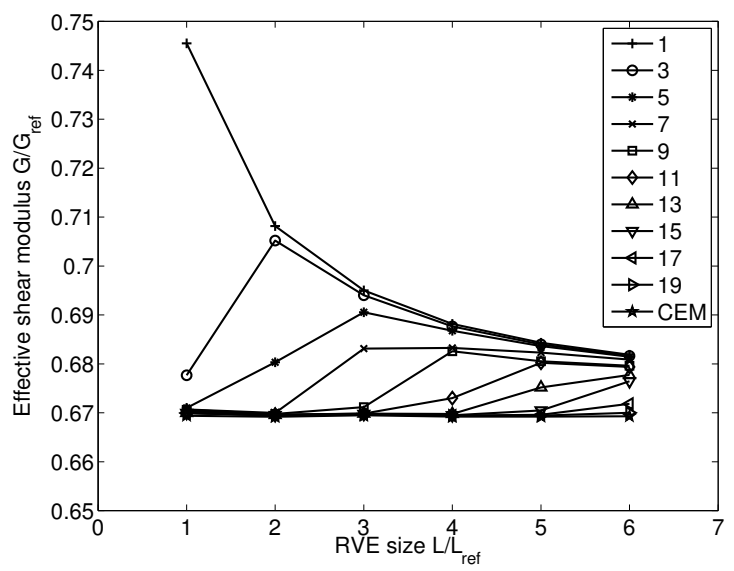

(b)

Figure 15: Influence of polynomial order for the Lagrange interpolation method, for a periodic micro-structure and (a) A periodic mesh and (b) A non-periodic mesh.

First the influence of the RVE size for periodic structures is studied for different interpolation orders when applying the periodic boundary conditions. 


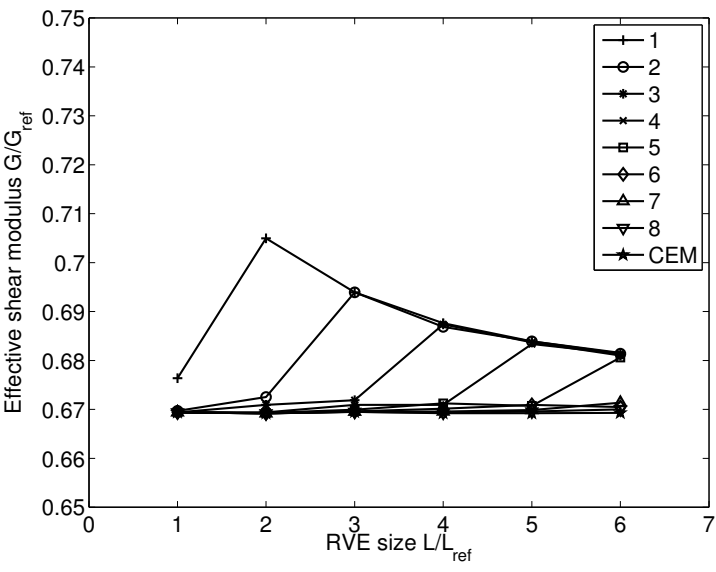

(a)

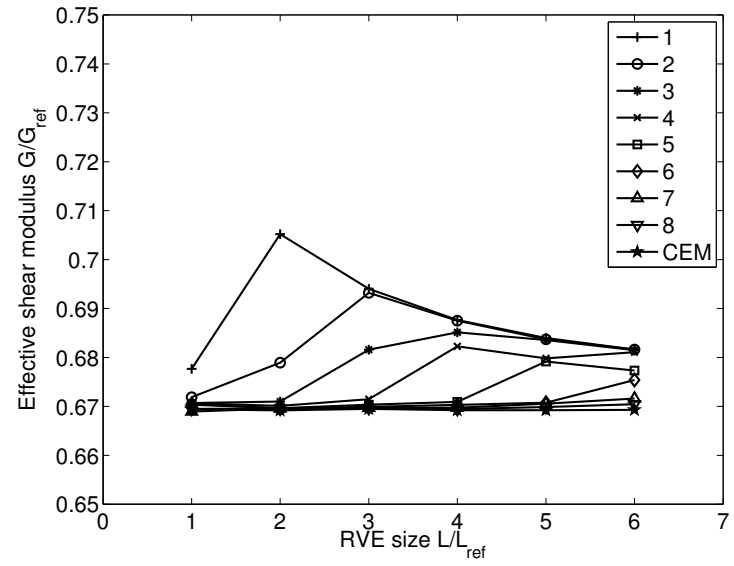

(b)

Figure 16: Influence of number of segments for the cubic spline interpolation method, for a periodic micro-structure and (a) A periodic mesh and (b) A non-periodic mesh.

Table 1: Selected polynomial order $n$ and number of segments $N$ for the micro-periodic structure.

\begin{tabular}{|c|c|c|c|c|c|c|}
\hline RVE size $\frac{L}{L_{\mathrm{ref}}}$ & 1 & 2 & 3 & 4 & 5 & 6 \\
\hline Polynomial order (Lagrange interpolation) $n$ & 5 & 7 & 11 & 13 & 17 & 19 \\
\hline Number of segments (cubic spline interpolation) $N$ & 2 & 4 & 5 & 6 & 7 & 8 \\
\hline
\end{tabular}

Fig. 15 shows the extracted in-plane shear modulus from the Lagrange interpolation method when increasing the polynomial order. The slight difference between results for the periodic meshes (Fig. 15a) and the non-periodic meshes (Fig. 15b) results from the finite element discretization only. Nevertheless, for both cases we can observe two bounds in terms of the RVE size for the effective shear modulus $G$ : (1) The upper bound is given by the linear interpolation (order $n=1$ ) and corresponds to the linear displacement boundary condition, and (2) lower bound is given by the periodic boundary condition applied with the constraint elimination method on a periodic mesh (CEM). All others values are within this domain, which converges toward the exact solution for increasing RVE size. Thus the Lagrange interpolation method also converges.

Fig. 16 represents the effective shear modulus extracted with the cubic spline interpolation method in terms of the RVE size and of the number of segments $N$. It is clear that the results given by $N=1$ are similar to the ones with the periodic mesh and with the non-periodic mesh given by the Lagrange interpolation method of order 3 . As for the Lagrange interpolation method, all the results obtained with periodic and non-periodic meshes by the cubic spline interpolation are within the two bounds presented above. This remark guarantees the method convergence.

On Figs. 15 and 16, it can be seen that the evolution of the effective properties is non monotonous. It can be explained by the poor resulting interpolation achieved when increasing the RVE size while keeping the polynomial order or number of segments constant. Moreover, there exists a polynomial order for the Lagrange interpolation and a number of segments for the spline interpolation, leading to results within $1 \%$ error to the results extracted with the exact periodic boundary condition (CEM). Table 1 shows a selection of these polynomial orders and number of segments for each RVE size, a larger RVE requires a larger order and a larger number of segments. Indeed, when the RVE size increases, in the context of the polynomial interpolation, a larger order or number of segments is required to capture the microscopic evolution of the boundary deformation. This table also shows that the optimal polynomial orders and number of segments are linear in function of the RVE size $\left(n \simeq 3 \frac{L}{L_{\mathrm{ref}}}+1\right.$ or $n \simeq 3 \frac{L}{L_{\mathrm{ref}}}+2$ to achieve odd number, and $N \simeq \frac{L}{L_{\mathrm{ref}}}+2$ ). For the selection of the parameters $n, N$ reported in Table 1, the relative error on the extracted in-plane shear modulus is shown in Fig. 17a. The reference effective shear computed in this case is 


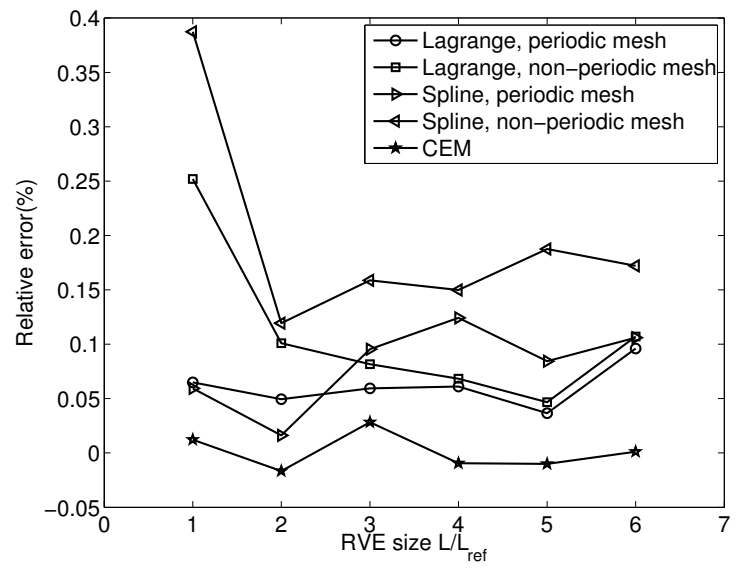

(a)

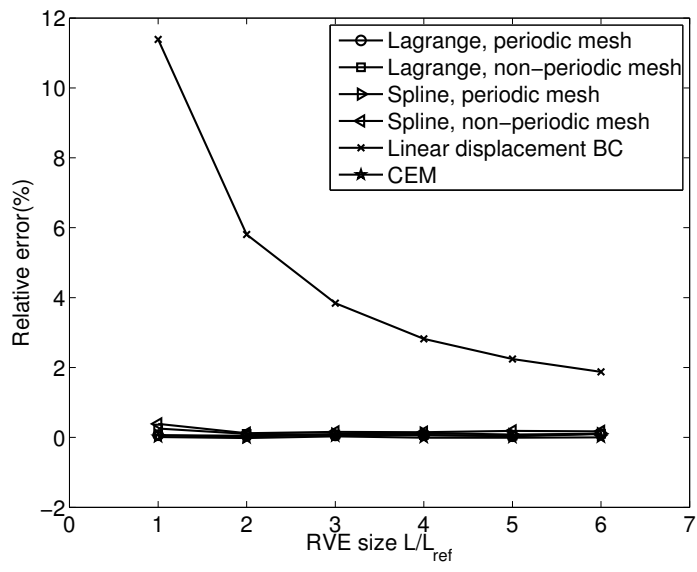

(b)

Figure 17: Relative error on the effective shear modulus: (a) Polynomial interpolation method and (b) Polynomial interpolation in comparison with the linear displacement boundary condition.

Table 2: Polynomial order $n$ and number of segments $N$ selected for the non-periodic micro-structure calculation.

\begin{tabular}{|c|c|c|c|c|c|c|c|}
\hline RVE size $\frac{L}{L_{\text {ref }}}$ & 1 & 2 & 3 & 4 & 5 & 6 & 7 \\
\hline Polynomial order (Lagrange interpolation) $n$ & 5 & 7 & 11 & 15 & 17 & 19 & 21 \\
\hline Number o segments (cubic spline interpolation) $N$ & 4 & 7 & 10 & 13 & 16 & 19 & 22 \\
\hline
\end{tabular}

18.0198GPa, and is well captured with the interpolation method. By comparison, the linear displacement boundary condition leads to error between 2 and $10 \%$ depending on the RVE size, see Fig. 17b.

\subsection{Non-periodic structures}

As previously said, for a reduced RVE size, the statistical properties of the RVE do not always correspond to the ones of the heterogeneous material. To satisfy a given accuracy requirement on the effective properties extraction,

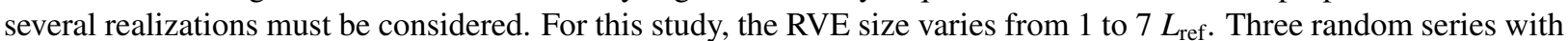
20 realizations are considered for the linear displacement boundary condition, for the periodic boundary condition enforced by the Lagrange interpolation and for the periodic boundary condition enforced by the cubic spline interpolation, respectively. The average void fraction is about 0.25 for all the series, although for a given RVE, the exact value cannot be 0.25 since it is generated automatically. In this study it is proposed to consider the polynomial order and number of segments reported in Table 2, which ensure the accuracy of the simulations. As for periodic microstructures, this table also shows that the optimal polynomial orders and number of segments are linear in function of the RVE size ( $n \simeq 3 \frac{L}{L_{\text {ref }}}+1$ or $n \simeq 3 \frac{L}{L_{\text {ref }}}+2$ to achieve odd number, and $\left.N \simeq 3 * \frac{L}{L_{\text {ref }}}+1\right)$

Effective in-plane shear modulus extracted for the different realizations are reported on Fig. 18, for the linear displacement boundary condition (Fig. 18a) and for the periodic boundary condition enforced using Lagrange interpolation and cubic spline interpolation methods (Fig. 18b-c). From these figures, it can be found that, the results converge when the RVE size increases despite the dispersion in results for small RVE sizes. Note that discrepancies between the results with the Lagrange interpolation method (Fig. 18b) and with the cubic spline interpolation method (Fig. 18c) result from the different geometry used for each realization. The average shear-modulus computed from realizations at a given RVE size is reported in Fig. 18d, for the different boundary conditions. For the Lagrange interpolation series, the dispersion of the void fraction and the dispersion of the computed effective shear modulus are presented via the accumulate distribution function (ADF) in Fig. 19 a and b respectively. This ADF is defined as

$$
\operatorname{ADF}(x)=P(X \leq x),
$$




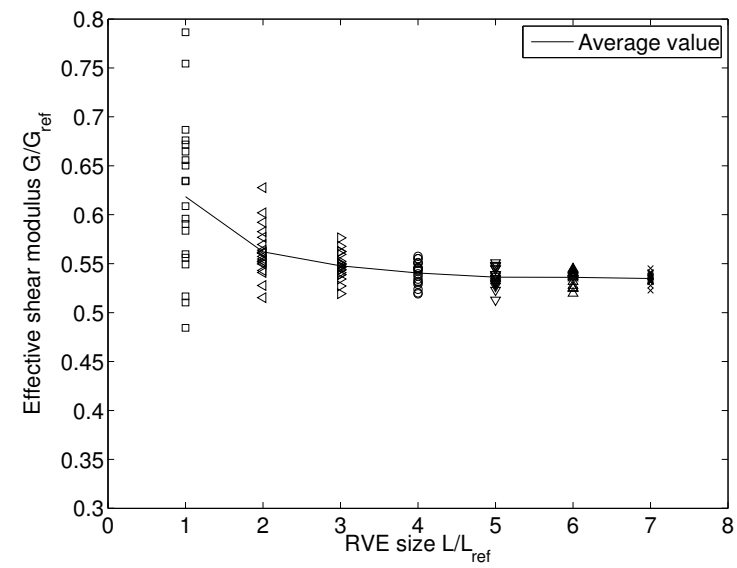

(a)

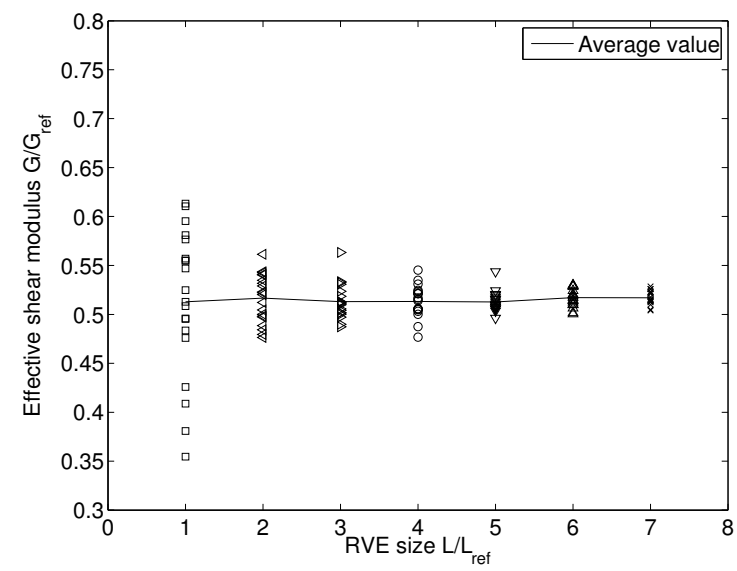

(c)

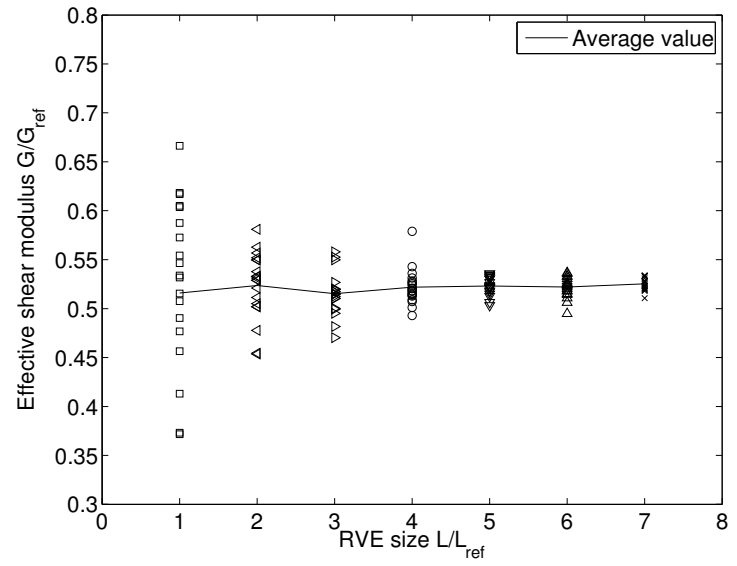

(b)

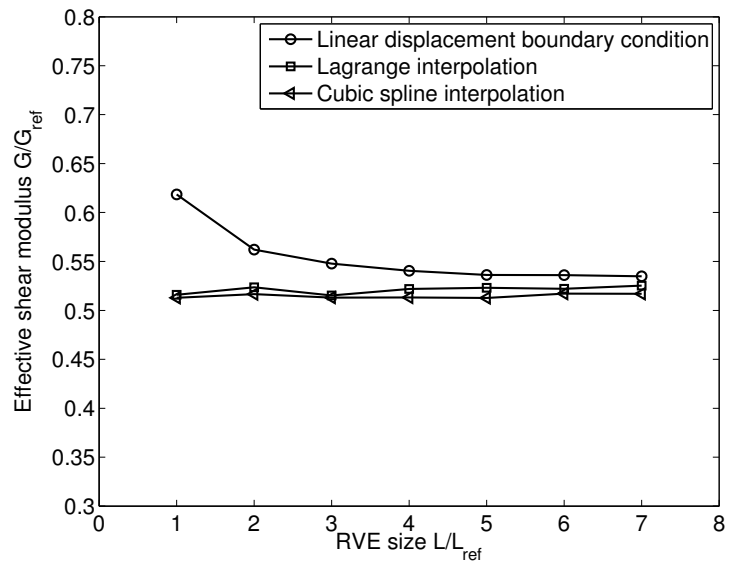

(d)

Figure 18: Effect of the RVE size for non-periodic micro-structures. (a) Linear displacement boundary condition. (b) Lagrange interpolation method for periodic boundary condition. (c) Cubic spline interpolation method for periodic boundary condition. Markers correspond to realization results, and the lines to the average value for a given RVE size. (d) Comparison of the average values

where the right-hand side represents the probability that the random variable $X$ takes on a value lower than or equal to $x$. It is found that the effective void fraction and shear modulus of the RVE are distributed around their average values. The smaller the RVE size, the more important the dispersion in the results. For a smaller RVE size, a larger number of realizations must then be used to capture the statistical properties of the micro-structures.

From this analysis, it can be concluded that, for non-periodic structures, even small RVE sizes can be considered if two conditions are respected: periodic boundary conditions are enforced and a reasonable number of realizations is performed.

\subsection{Computational efficiency}

To demonstrate the efficiency of the polynomial interpolation method, a summary of the number of dofs and the computational time required to solve the linear system is presented in Table 3 . A RVE size $6 L_{\text {ref }}$ of the periodic structure and meshed with a periodic mesh is considered. The results are given for the linear displacement boundary condition, for periodic boundary condition enforced with the classical constraint elimination method, and for periodic boundary condition enforced with Lagrange and cubic spline formulations. Table 3 shows that the polynomial interpolation method can reduce the number of dofs as compared with the CEM, but increases slightly the computational time to solve the linear system due to the increase in the bandwidth of the stiffness matrix. However the use of the 


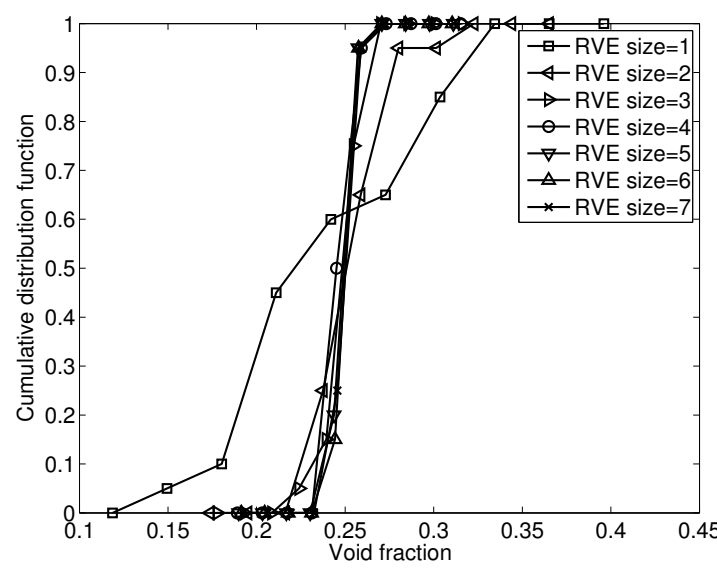

(a)

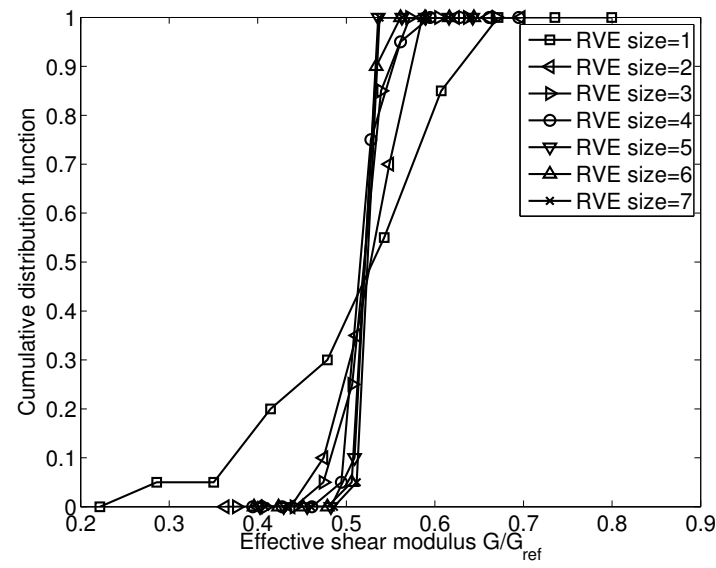

(b)

Figure 19: Cumulative distribution function for non-periodic structures computed with periodic boundary condition and Lagrange interpolation: (a) Void fraction and (b) In-plane effective shear modulus.

Table 3: Computational time and number of degrees of freedom (dofs) (RVE size of $6 L_{\mathrm{ref}}$ of the periodic structures and periodic mesh)

\begin{tabular}{|l|c|c|c|c|}
\hline Method & Linear displacement & CEM & Lagrange (degree 19) & Cubic spline (8 segments) \\
\hline Number of dofs & 28410 & 28880 & 28484 & 28476 \\
\hline $\begin{array}{l}\text { Time to solve the } \\
\text { linear system (s) }\end{array}$ & 3.02 & 3.19 & 3.71 & 3.28 \\
\hline $\begin{array}{l}\text { Effective shear } \\
(\mathrm{GPa})\end{array}$ & 18.36 & 18.02 & 18.04 & 18.04 \\
\hline
\end{tabular}

polynomial interpolation method leads to more accurate results than the the linear displacement method, allowing reducing the RVE size, and thus the real computation time.

\section{Implementation for three-dimensional cases}

In this section, the efficiency of the polynomial interpolation method, as a way of enforcing the periodic boundary condition, is demonstrated for the 3-dimensional case. The Lagrange interpolation and the cubic spline interpolation are used as a basis for the Coons interpolation presented in section 3.2.

First, to validate the approach with respect to the constraint elimination method, a periodic structure with a periodic mesh is considered. The three-dimensional RVE, which contains 8 spherical voids periodically distributed, whose size is $L / L_{\text {ref }}=2$ and whose void fraction is 0.27 is presented in Fig. 20. The RVE material is elastic: elastic modulus $E=70 \mathrm{GPa}$ and Poisson ratio $v=0.3$. The macro-strain tensor applied to the RVE implies different loading modes:

$$
\bar{\varepsilon}=\left[\begin{array}{ccc}
0.01 & 0.005 & 0.005 \\
0.005 & 0.01 & 0.005 \\
0.005 & 0.005 & 0.01
\end{array}\right]
$$

Three ways of enforcing the periodic boundary condition are successively applied: first the CEM, second the Lagrange interpolation method with a polynomial order of 5 for all edges of the RVE, and third the cubic spline interpolation with 5 segments on each edge of RVE. The average macro-stress tensor computed by using the volume integration 


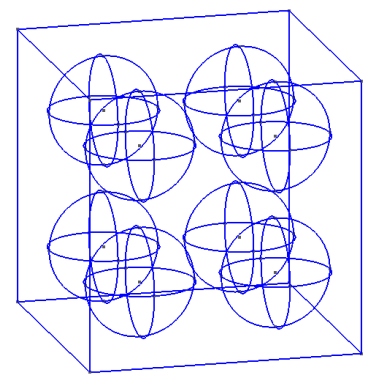

(a)

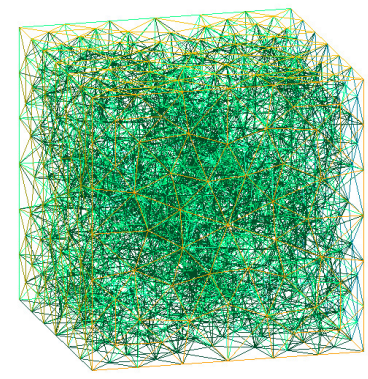

(b)

Figure 20: Three-dimensional periodic RVE: (a) Geometry and (b) Periodic mesh.

formula (23) for the three methods are respectively

$$
\begin{aligned}
\bar{\sigma}_{\text {CEM }} & =\left[\begin{array}{ccc}
984.801 & 164.761 & 165.98 \\
164.761 & 985.002 & 164.653 \\
165.98 & 164.653 & 984.876
\end{array}\right] \mathrm{MPa}, \\
\bar{\sigma}_{\text {Lagrange }} & =\left[\begin{array}{ccc}
988.71 & 167.202 & 168.302 \\
167.202 & 988.812 & 167.646 \\
168.302 & 167.646 & 988.572
\end{array}\right] \mathrm{MPa}, \text { and } \\
\bar{\sigma}_{\text {spline }} & =\left[\begin{array}{lll}
986.437 & 166.003 & 167.221 \\
166.003 & 986.534 & 166.254 \\
167.221 & 166.254 & 986.341
\end{array}\right] \mathrm{MPa} .
\end{aligned}
$$

Results obtained with the three methods to enforce the PBCs: CEM, Lagrange, and cubic spline interpolations are illustrated in Fig. 21 in terms of the von Mises stress

$$
\begin{aligned}
\sigma_{V M} & =\sqrt{3 J_{2}}, \text { with } \\
J_{2} & =\frac{1}{2} s_{i j} s_{i j}, \text { and } \\
s_{i j} & =\sigma_{i j}-\frac{1}{3} \delta_{i j} \sigma_{k k},
\end{aligned}
$$

where $\sigma_{i j}$ is the Cauchy stress tensor, and $s_{i j}$ is the deviatoric tensor. It can be seen in Fig. 21 that the results obtained with the interpolation methods are good agreement with the CEM solution.

For non-periodic structures, for which a periodic mesh is impossible to generate, a non-periodic RVE of size $L / L_{\text {ref }}=2.5$ and whose void fraction is 0.41 is studied as presented in Fig. 22a. Cavities are cylindrical and unidirectional. The generated mesh is non-periodic and is shown in Fig. 22a. The macro-strain tensor applied to the RVE implies different loading modes:

$$
\overline{\boldsymbol{\varepsilon}}=\left[\begin{array}{ccc}
0.01 & 0.005 & 0.005 \\
0.005 & 0.01 & -0.005 \\
0.005 & -0.005 & -0.01
\end{array}\right]
$$

The material is also elastic (elastic modulus $E=70 \mathrm{GPa}$ and Poisson ration $v=0.3$ ). As the CEM solution cannot be reached, the Lagrange interpolation method is used with a polynomial order of 15 for all edges of the RVE and the 


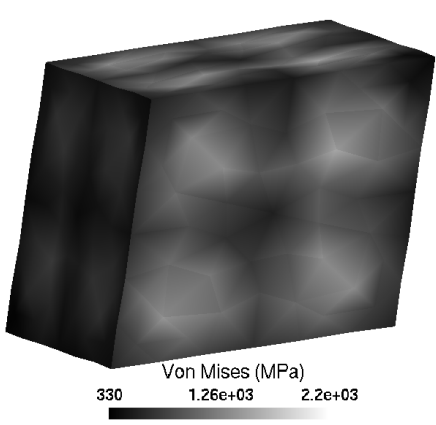

(a)

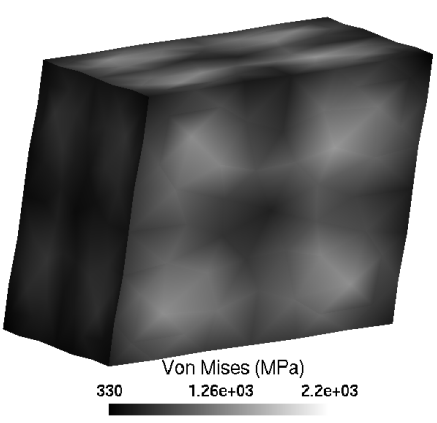

(b)

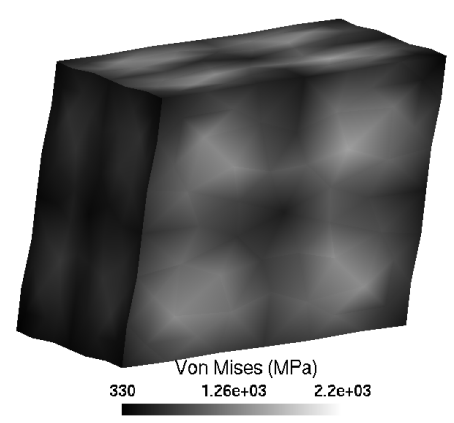

(c)

Figure 21: Distribution of the von Mises stress on the periodic RVE with: (a) CEM to enforce the periodic boundary condition (b) Lagrange interpolation to enforce the periodic boundary condition (c) Cubic spline interpolation to enforce the periodic boundary condition.

cubic spline interpolation is implemented with 10 segments on each edge of RVE.

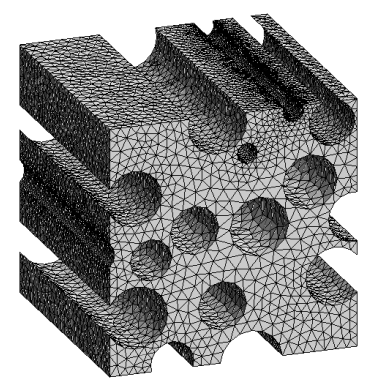

(a)

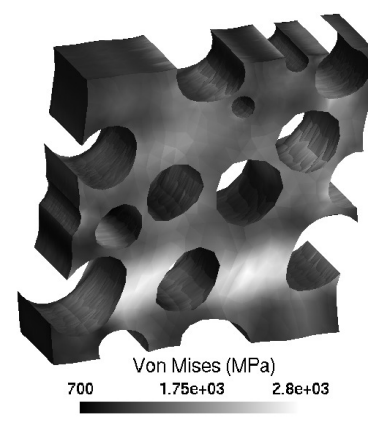

(b)

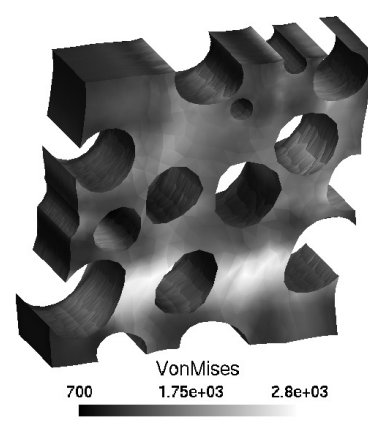

(c)

Figure 22: Equivalent von Mises stress in the non-periodic RVE with the periodic boundary condition enforced with the polynomial interpolation: (a) 3D mesh, (b) Lagrange interpolation and (c)Cubic spline interpolation.

The resulting von Mises stress of the RVE are presented in Figs. 22b and c for the Lagrange interpolation and the cubic spline interpolation respectively. This figure shows that the results obtained with the two approaches are in good agreement. The average macro-stress tensor can be also computed by using the volume integration formula (23). The average macro-stress tensor obtained with the Lagrange interpolation is given by

$$
\bar{\sigma}_{\text {Lagrange }}=\left[\begin{array}{ccc}
281.583 & 92.392 & 121.181 \\
92.392 & 270.111 & -115.835 \\
121.181 & -115.835 & -247.78
\end{array}\right] \mathrm{MPa}
$$

while the one obtained using the cubic spline interpolation is given by

$$
\bar{\sigma}_{\text {spline }}=\left[\begin{array}{ccc}
281.399 & 91.9833 & 121.239 \\
91.983 & 268.85 & -115.614 \\
121.239 & -115.614 & -248.214
\end{array}\right] \mathrm{MPa} .
$$

This section shows that the polynomial interpolation method can be used to apply the periodic boundary condition on a 3D-RVE in a straightforward way for both periodic meshes and non-periodic meshes. 


\section{Conclusions}

In this paper, a new method to enforce the periodic boundary condition on arbitrary finite element meshes by using the polynomial interpolation is presented. The polynomial interpolation is carried out by using either the Lagrange interpolation formulation or the cubic spline interpolation formulation. This method is suitable for general mesh design, periodic or non-periodic meshes, in the 2-dimensional case and in the 3-dimensional case. A key advantage of this method is the elimination of the need of matching nodes usually required when applying the periodic boundary condition.

To illustrate the method capabilities, two kinds of micro-structures are considered. One is a periodic distribution of holes and another one is a random distribution of holes. While the meshes generated for the periodic micro-structures are controllable, the meshes generated for the non-periodic micro-structures are not totally controlled. In the first case the periodic boundary condition can be applied by traditional constraint elimination method or by the newly proposed polynomial interpolation method, which allows validating our approach. In the second case, only the new method can be considered, which allows demonstrating its versatility.

The influence of the polynomial order of the interpolation is then studied. The parameter of the Lagrange interpolation is the polynomial order and the parameter of the cubic spline interpolation is the number of segments. The selection of these parameters is not trivial, as a larger RVE needs a larger polynomial order or a larger number of segments to reach a given accuracy.

However, for a given RVE size, the results always converge with the increase of the polynomial order and the polynomial interpolation is shown to provide accurate results in comparison to those given by the constraint elimination method. In all the cases, the method is more accurate than the enforcement of linear displacement for boundary conditions, which is usually considered for non-periodic micro-structures. Moreover, the presented formulation does not induce a sensitive increase in the computational expenses as the number of degrees of freedom is not increased.

\section{Acknowledgment}

Les recherches ont été financées grâce à la subvention "Actions de recherche concertées ARC 09/14-02 BRIDGING - From imaging to geometrical modelling of complex micro structured materials: Bridging computational engineering and material science" de la Direction générale de l'Enseignement non obligatoire de la Recherche scientifique, Direction de la Recherche scientifique, Communauté française de Belgique, et octroyées par l'Académie Universitaire Wallonie-Europe.

\section{Appendix A. Generation of randomly distributed hole structures}

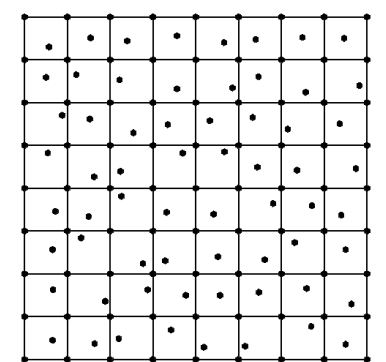

(a)

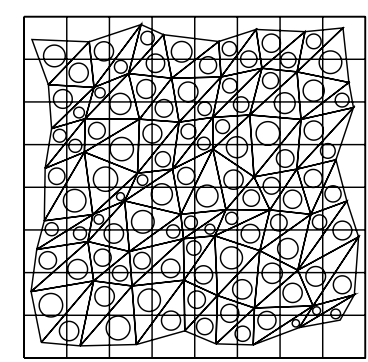

(b)

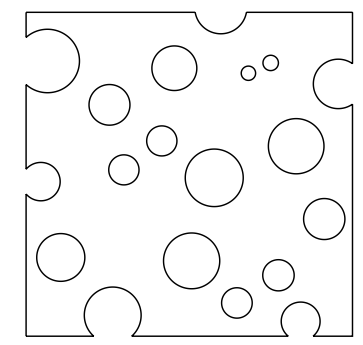

(c)

Figure A.23: Generation of a non-periodic structure with randomly distributed holes. (a) Random set of points generated inside the square cells, $L_{\text {ref }}$ is size of each cell. (b) Triangles generation from a this set of points and circles generation from these triangles. Each circle center coincides to the in-circle center of the triangles. Porosity is controlled by the circle radius. (c) A RVE cut from the hole structure with size $L / L$ ref $=3$. 
The generation of a non-periodic structure with randomly distributed holes is based on the approach of Cui and O'Sullivan [19]. A random set of points is generated inside a square-cells system as presented in Fig. A.23a. Each point of this set is normally distributed on each cell of size $L_{\text {ref }}$. From this set of points, a set of triangles is created as presented on Fig. A.23b. In each triangle, a circle, whose center is the in-circle center of the triangle, is created to model the void in the material. The circle radius $r$ is constrained to be lower than the in-circle radius $r_{\text {in }}$ by a user factor $\alpha$ such that $r=\alpha r_{\text {in }}$. The structure porosity is controlled by the factor $\alpha$. Factor of 0.0 leads to a structure without voids and of 1.0 leads to highest possible porosity. The RVE is cut from a larger created structure, as presented on Fig. A.23c. In this example, the RVE size is $L=3 L_{r e f}$, and $\alpha=0.7$. Void fraction is controlled by the parameter $\alpha$, such that

$$
f=\frac{\sum_{i} \pi r_{i}^{2}}{L^{2}}=\frac{\sum_{i} \pi\left(\frac{r_{i}}{L_{\mathrm{ref}}}\right)^{2}}{\left(\frac{L}{L_{\mathrm{ref}}}\right)^{2}},
$$

where $r_{i}$ is the radius of void $i$ and $L$ is the RVE size.

\section{References}

[1] O. Pierard, C. Friebel, I. Doghri, Mean-field homogenization of multi-phase thermo-elastic composites: a general framework and its validation, Composites Science and Technology 64 (10-11) (2004) 1587-1603. doi:DOI: 10.1016/j.compscitech.2003.11.009.

URL http: //www.sciencedirect.com/science/article/B6TWT-4BJX082-3/2/79e5fb931d7e0e48e9c9d6c2f4c11df5

[2] J. Michel, H. Moulinec, P. Suquet, Effective properties of composite materials with periodic microstructure: a computational approach, Computer Methods in Applied Mechanics and Engineering 172 (1-4) (1999) 109 - 143. doi:10.1016/S0045-7825(98)00227-8. URL http: //www.sciencedirect.com/science/article/pii/S0045782598002278

[3] C. Miehe, A. Koch, Computational micro-to-macro transitions of discretized microstructures undergoing small strains, Archive of Applied Mechanics 72 (4) (2002) 300-317, 10.1007/s00419-002-0212-2. URL http://dx.doi .org/10.1007/s00419-002-0212-2

[4] V. Kouznetsova, W. A. M. Brekelmans, F. P. T. Baaijens, An approach to micro-macro modeling of heterogeneous materials, Computational Mechanics 27 (1) (2001) 37-48.

URL http://dx.doi.org/10.1007/s004660000212

[5] V. Kouznetsova, M. G. D. Geers, W. A. M. Brekelmans, Multi-scale constitutive modelling of heterogeneous materials with a gradientenhanced computational homogenization scheme, Int. J. Numer. Meth. Engng. 54 (8) (2002) 1235-1260. URL http://dx.doi.org/10.1002/nme.541

[6] V. G. Kouznetsova, M. G. D. Geers, W. A. M. Brekelmans, Multi-scale second-order computational homogenization of multi-phase materials: a nested finite element solution strategy, Computer Methods in Applied Mechanics and Engineering 193 (48-51) (2004) 5525-5550, advances in Computational Plasticity. doi:DOI: 10.1016/j.cma.2003.12.073.

URL http://www.sciencedirect.com/science/article/B6V29-4D4D4JV-3/2/6fd8ac06299b9a26d8a17438871de868

[7] L. Kaczmarczyk, C. J. Pearce, N. Bićanić, Scale transition and enforcement of rve boundary conditions in second-order computational homogenization, Int. J. Numer. Meth. Engng. 74 (3) (2008) 506-522. URL http://dx.doi.org/10.1002/nme.2188

[8] P. Kanoute, D. Boso, J. Chaboche, B. Schrefler, Multiscale methods for composites: A review, Archives of Computational Methods in Engineering 16 (1) (2009) 31-75, 10.1007/s11831-008-9028-8. URL http://dx.doi .org/10.1007/s11831-008-9028-8

[9] D. Peric, E. A. de Souza Neto, R. A. Feijóo, M. Partovi, A. J. C. Molina, On micro-to-macro transitions for multi-scale analysis of non-linear heterogeneous materials: unified variational basis and finite element implementation, Int. J. Numer. Meth. Engng. URL http://dx.doi.org/10.1002/nme.3014

[10] M. G. D. Geers, V. G. Kouznetsova, W. A. M. Brekelmans, Multi-scale computational homogenization: Trends and challenges, Journal of Computational and Applied Mathematics 234 (7) (2010) 2175-2182, fourth International Conference on Advanced COmputational Methods in ENgineering (ACOMEN 2008). doi:DOI: 10.1016/j.cam.2009.08.077.

URL http://www. sciencedirect.com/science/article/B6TYH-4X1J73B-8/2/ee8d9b69133503eaf14b00ddbe1bd8f5

[11] T. Kanit, S. Forest, I. Galliet, V. Mounoury, D. Jeulin, Determination of the size of the representative volume element for random composites: statistical and numerical approach, International Journal of Solids and Structures 40 (13-14) (2003) 3647-3679. doi:DOI: 10.1016/S00207683(03)00143-4

URL http://www.sciencedirect.com/science/article/B6VJS-48GDV9K-1/2/2dd6cda9c49706ed2c969bdea531bb50

[12] K. Terada, M. Hori, T. Kyoya, N. Kikuchi, Simulation of the multi-scale convergence in computational homogenization approaches, International Journal of Solids and Structures 37 (16) (2000) 2285-2311. doi:DOI: 10.1016/S0020-7683(98)00341-2. URL http://www.sciencedirect.com/science/article/B6VJS-3YDFYCH-4/2/788d085d620c6c5ae804d8c4e4f8ae9b

[13] F. Larsson, K. Runesson, S. Saroukhani, R. Vafadari, Computational homogenization based on a weak format of micro-periodicity for rveproblems, Computer Methods in Applied Mechanics and Engineering 200 (1-4) (2011) 11-26. doi:DOI: 10.1016/j.cma.2010.06.023. URL http: //www.sciencedirect.com/science/article/B6V29-50J9GSY-1/2/50ad840717a7cc397ab171da344df990 
[14] G. Legrain, P. Cartraud, I. Perreard, N. Moës, An x-fem and level set computational approach for image-based modelling: Application to homogenization, Int. J. Numer. Meth. Engng.

URL http://dx.doi.org/10.1002/nme.3085

[15] Z. Yuan, J. Fish, Toward realization of computational homogenization in practice, Int. J. Numer. Meth. Engng. 73 (3) (2008) $361-380$. URL http://dx.doi.org/10.1002/nme.2074

[16] J. M. Tyrus, M. Gosz, E. DeSantiago, A local finite element implementation for imposing periodic boundary conditions on composite micromechanical models, International Journal of Solids and Structures 44 (9) (2007) 2972-2989. doi:DOI: 10.1016/j.ijsolstr.2006.08.040. URL http: //www.sciencedirect.com/science/article/B6VJS-4KW5FH7-2/2/5ff1cecbcb5cdcf33cab7c7f6f6a1935

[17] C. Geuzaine, J.-F. Remacle, Gmsh: A 3-d finite element mesh generator with built-in pre- and post-processing facilities, International Journal for Numerical Methods in Engineering 79 (11) (2009) 1309-1331. URL http://dx.doi.org/10.1002/nme.2579

[18] C. Pelissou, J. Baccou, Y. Monerie, F. Perales, Determination of the size of the representative volume element for random quasi-brittle composites, International Journal of Solids and Structures 46 (14-15) (2009) 2842 - 2855. doi:DOI: 10.1016/j.ijsolstr.2009.03.015. URL http: //www.sciencedirect.com/science/article/pii/S0020768309001334

[19] L. Cui, C. O'Sullivan, Analysis of a triangulation based approach for specimen generation for discrete element simulations, Granular Matter 5 (2003) 135-145, 10.1007/s10035-003-0145-7.

URL http: //dx.doi.org/10.1007/s10035-003-0145-7 